\title{
A Survey of Geotechnical System Identification Techniques
}

\author{
Caglar Oskay ${ }^{*}$ and Mourad Zeghal,
}

\begin{abstract}
Identification and inverse problem techniques play an important role in the characterization and modeling of geotechnical systems. These techniques have been used in estimation of system parameters, model development and calibration, as well as simulation of earthquake ground motions. The recent availability of high quality seismic records of sites equipped with downhole accelerometer arrays led to a burgeoning of identification and inverse problem studies involving geotechnical systems. This paper presents a survey of system identification techniques and analyses of full- and small-scale soil systems, with an emphasis on geotechnical earthquake engineering problems.
\end{abstract}

\section{Introduction}

Geotechnical structures and soil deposits exhibit a broad range of response patterns when subjected to dynamic excitations. The response of these systems depends on soil stiffness properties, degree of saturation and pore water pressure, stratigraphy, topography, and other factors. The usual forward boundary-value-problem analysis requires the availability of an analytical or a computational model of the system and a full set of associated parameters. This model and parameters are then used to predict the system response to external loading conditions.

Soil-sample experiments (e.g., triaxial tests) have been widely used to evaluate parameters and calibrate models of geotechnical systems. Because of limitations in reproducing the complex in-situ stress and pore fluid conditions, the consensus is that these tests may not fully reflect reality. Seismic records and experimental results of full- and small-scale tests are a unique source of information which complement knowledge derived from soil-sample testing. However, thorough monitoring of the seismic excitation and associated response of massive geotechnical systems is a significant technical challenge. Historically, only a limited number of sensors have been used to monitor these systems.

System identification and inverse problem analyses play an important role in development, validation and calibration of soil models, as well as estimation of in-situ properties and parameters, using experimental and observational earthquake data. The literature pertaining to geotechnical-system identification using seismic records is rather scarce, compared for instance to structural identifications. This limited activity reflects the problem complexity and a historical lack of data sets suited to inverse problem analyses in geotechnical earthquake engineering. The recent availability of relatively high quality

${ }^{*}$ Dept. of Civ. and Env. Engrg., Vanderbilt University, VU Station B\#351831, 2301 Vanderbilt Place, Nashville, TN 37067. E-mail: caglar.oskay@ @anderbilt.edu.

${ }^{\dagger}$ Dept. of Civ. and Env. Engrg., Rensselaer Polytechnic Institute, 110 Eighth Str., Troy, NY 12180. E-mail: zeghal@rpi.edu. 
downhole array seismic records of sites in the USA, Mexico, Japan and Taiwan led to a burgeoning of research efforts addressing the identification of site dynamic response characteristics. Elgamal et al. [24] and Zeghal and Elgamal [105] presented a comprehensive list of sites equipped with downhole array instrumentation along with an overview of associated identification studies. Previously, Glaser [40] summarized a number of identification techniques used to estimate soil properties from strong motion recordings. In contrast, a relatively significant number of review articles were published in the field of structural engineering [9, 37, 45, 48, 50, 55, 86]. Extensive research and analyses on relevant system identification topics have been conducted in other fields of engineering and sciences such as electrical engineering, automation, geophysics, and other closely related areas. This is reflected in a substantial number of overview papers, some of which focused on specific topics, such as Bayesian analyses [77], non-parametric identification techniques [97] and system identification of distributed parameter systems $[62,82,96]$. A number of published books, monographs and conference proceedings $[5,10,14,34,51,52,61,67,71,78,92,94]$ also covered these topics, along with other system identification subjects.

This paper presents a review of system identification techniques and analyses of full- and smallscale geotechnical earthquake engineering systems. The surveyed studies were categorized based on the characteristics of involved models (Fig. 1). The following sections provide an assessment of: (1) response characterization and pattern recognition studies, (2) time series analyses, and (3) identifications involving constitutive (stress-strain) modeling.

\section{Characterization and Pattern Recognition of System Response}

Pattern recognition analyses generally consist of identifications aimed at characterizing and assessing the nature of system response and loading conditions. Simple operations are performed on measured output (response) and possibly input (excitation) in order to gain insight into the system properties and response mechanisms without recourse to explicit modeling of the involved physical phenomena. These operations are diverse and range from simple spectral ratio analyses to stress-strain imaging for statically determinate systems (Fig. 1). Information derived from system characterization studies have been used in modeling and parameterization of the involved phenomena.

\subsection{Transfer function and spectral analyses}

Transfer functions, or ratios of the Fourier amplitude spectra of input and output acceleration pairs, have been widely employed to estimate natural frequencies of vibrations and associated (shear and normal) wave propagation velocities of sites, earth dams, and other systems [3, 12, 23, 39, 49, 89, 99]. Spectral ratios are adequate in analyses of system response to non-destructive forced-vibrations, as well as low and moderate amplitude earthquakes, in which stiffness reductions are insignificant. Slight modifications are required to analyze large amplitude motions accompanied by nonlinearities and softening of soil materials. For instance, transfer functions may be computed for successive or overlapping time windows which have relatively little variations in acceleration amplitude. Chang et al. [17] used such a technique to estimate shear wave velocities of the Lotung (Taiwan) site using acceleration time-histories of the Large Scale Seismic Test (LSST) downhole array [93]. For any two-strata system of soil comprised between a surface and two consecutive downhole accelerometers (Fig. 2), the average fundamental frequency, $f_{A}$, of the upper soil layer $A$ and the system composite fundamental frequency, $f$, were estimated using transfer functions. The fundamental frequency, $f_{B}$, of 


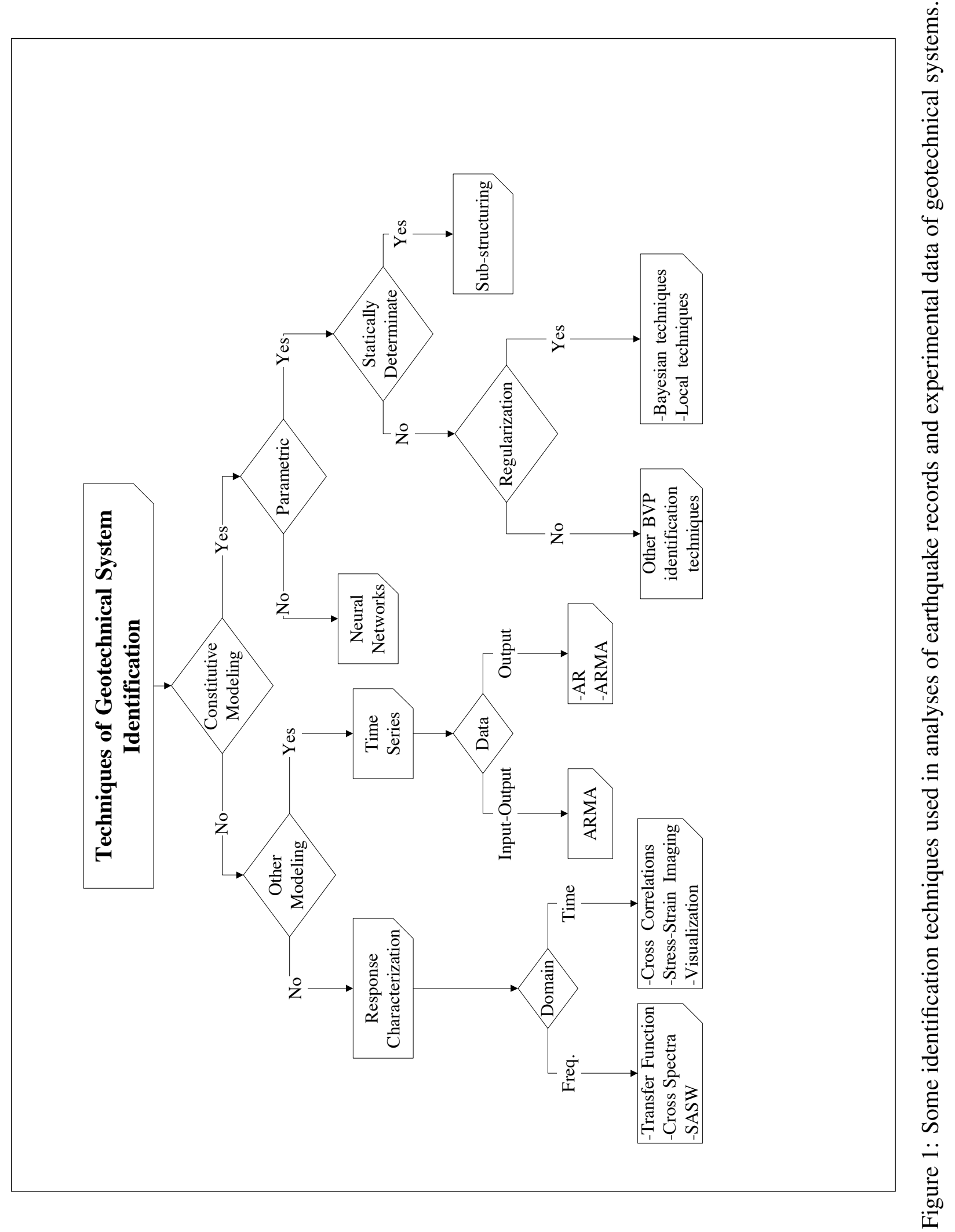




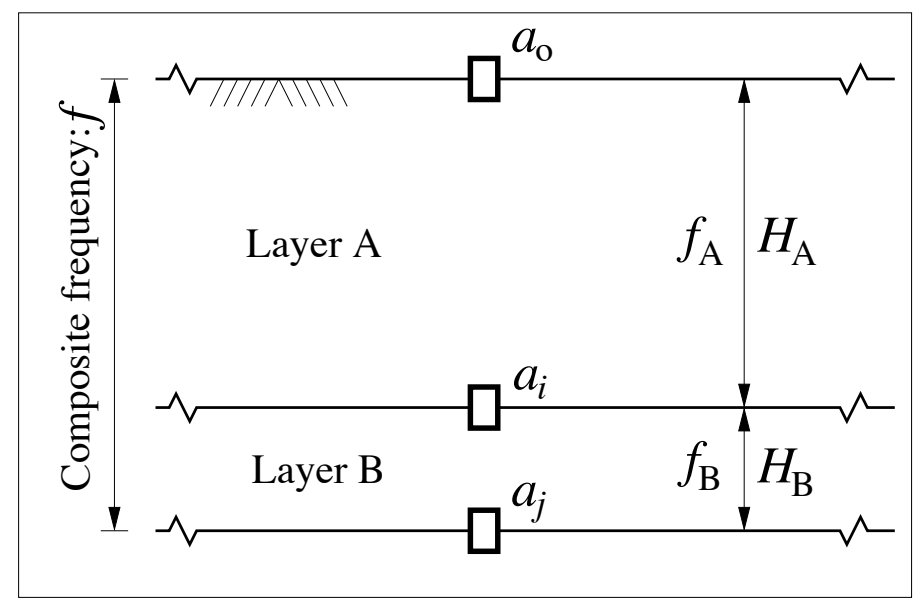

Figure 2: Schematic of a 2-layer soil profile used in transfer function analyses.

the underlying layer $B$ was then computed using the following implicit wave propagation equation [66]:

$$
\tan \left[\left(\frac{\pi}{2} \frac{f}{f_{A}}\right)\left(\frac{\pi}{2} \frac{f}{f_{B}}\right)\right]=\frac{\rho_{B}}{\rho_{A}} \frac{H_{B}}{H_{A}} \frac{f_{B}}{f_{A}}
$$

in which $\rho_{A}$ and $\rho_{B}$ are respectively mass densities of layers $A$ and $B$, and $H_{A}$ and $H_{B}$ are layer thicknesses (Fig. 2). The associated shear wave velocities were evaluated using: $V_{A}=4 H_{A} f_{A}$ and $V_{B}=4 H_{B} f_{B}$. These velocities and the equivalent linear program Shake were subsequently used to estimate the shear modulus reduction curves of soil strata at Lotung site (Fig. 3). Wen [98] also used this spectral ratio method to study the Lotung LSST data and concluded that the response of this site was marked by strong nonlinearities during earthquakes with peak ground accelerations larger than approximately $0.15 \mathrm{~g}$. Zorapapel and Vucetic [114] employed transfer functions of the 1987 Wildlife Refuge seismic records to assess the relationship between lengthening of site fundamental period, amplification factors and excess pore pressure build-up. Ghayamghamian and Motosaka [39] employed a time-window analysis to assess the decrease in fundamental resonant frequency, $f$, with increase in acceleration amplitudes at Chiba (Japan) site. Normalized shear modulus reduction curves of surfacial soil strata were obtained using; $\left(G / G_{o}\right)=\left(f / f_{o}\right)^{2}$, in which $f_{o}$ is low-amplitude shaking resonant frequency. Finite differentiation schemes [104] were employed to estimate the associated strains. The transfer function analysis based on downhole acceleration data may lead to erroneous results due to wave reflections at the ground surface and layer interfaces. A study by Safak [84] to assess the adequacy of transfer functions concluded that the cross-spectral ratios approach provides probabilistically more reliable estimates than the simple transfer function technique. Safak suggests that spectral ratios should not be used to determine site amplification from downhole accelerations.

A cross-spectral analysis was used by Elgamal, Zeghal and co-workers [30] to identify the modal configurations of level sites equipped with dense vertical (downhole) arrays such as Lotung. The amplitude of cross-spectrum energy function, $s_{i j}(f)$, of two downhole array acceleration records $a_{i}$ and $a_{j}$ (as shown in Fig. 2), peaks at resonant and input frequencies. A peak represents resonance if the corresponding phase angle approaches $0^{\circ}$ or $180^{\circ}$. The amplitude of the $k^{\text {th }}$ modal configuration $\phi_{k}$ (of frequency $f_{k}$ ) at the $i^{\text {th }}$ instrument station (at a depth $z_{i}$ ) may then be computed using:

$$
\phi_{k}\left(z_{i}\right)= \pm \sqrt{s_{i i}\left(f_{k}\right)}
$$

in which $s_{i i}$ is auto-spectral density function amplitude of $a_{i}$. 

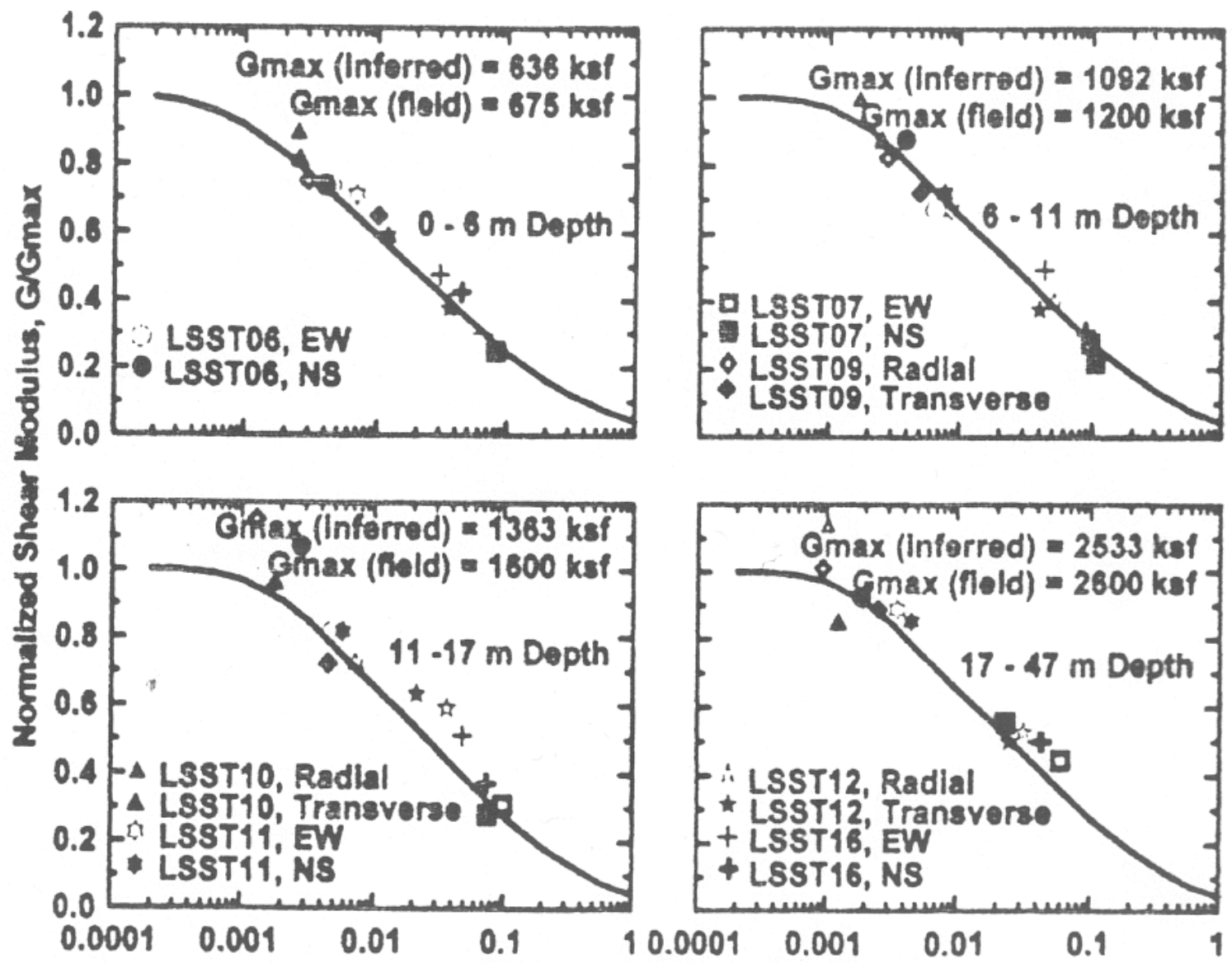

Effoctlve Shear Strain (\%)

Figure 3: Shear modulus reduction curves of Lotung site evaluated using transfer function analyses (by Chang et al. [17]). 


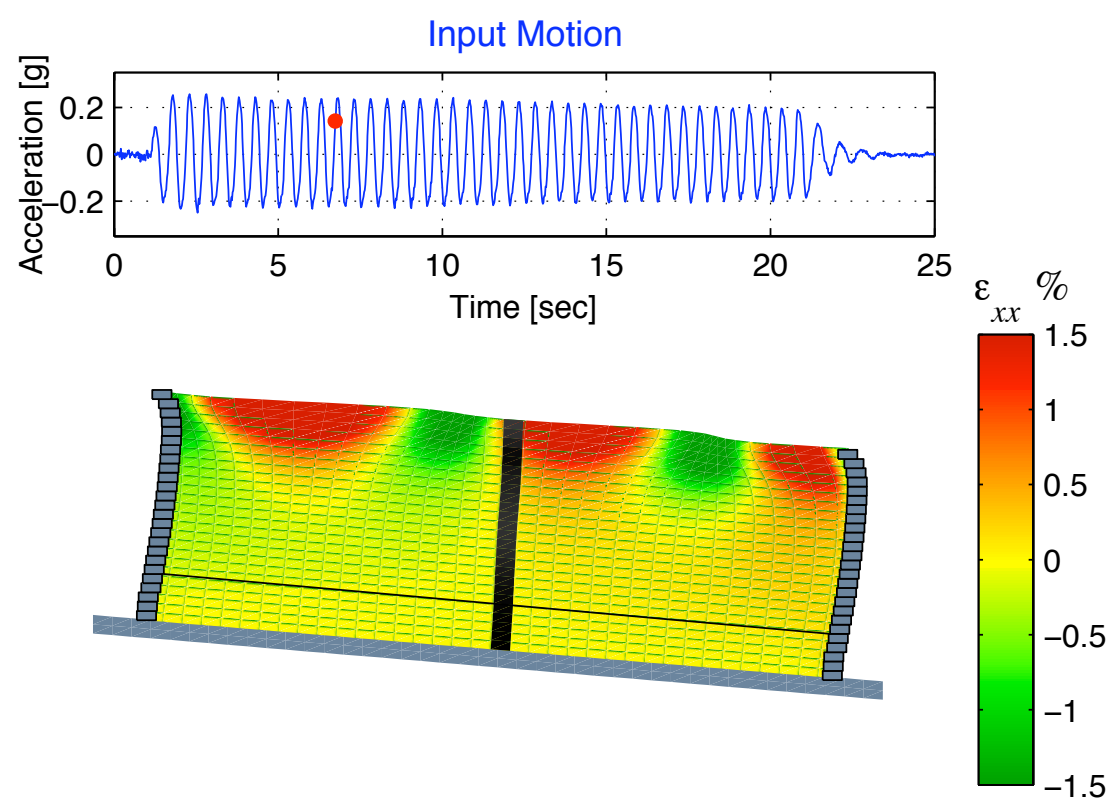

Figure 4: Displacement and lateral normal strain field of a pile-soil system as identified using spectral interpolations by Zeghal et al. [109].

\subsection{Spectral interpolations and visualization}

As mentioned above, a limited number of sensors is commonly used to monitor the response of distributed parameter geotechnical systems. The information provided by these sensors is often patchy and interpolations are necessary to assess the totality of a system response. Zeghal, Oskay and coworkers [111, 109] used global interpolations to reconstitute the displacement and deformation fields of centrifuge models of soil systems:

$$
\mathbf{u}(\mathbf{x}, t)=\sum_{i=1}^{n} \alpha_{i}(t) \phi_{i}(\mathbf{x}, t)
$$

in which $\mathbf{u}(\mathbf{x}, t)$ is displacement as a function of space, $\mathbf{x}$, and time, $t$, coordinates, $\phi_{i}$ are a set of interpolation (shape) functions that satisfy the system kinematic boundary conditions, $\alpha_{i}$ are generalized coordinates, and $n$ is number of used functions. The modal configurations of the linearized vibrations were used as shape functions, and were adaptively varied in time to reflect any changes in system stiffness properties. These changes were assessed using cross-correlations of recorded accelerations, and the generalized coordinates $\alpha_{i}$ were identified using least squares optimizations of discrepancies between recorded and computed (Eq. 3) motions. Figure 4 displays the optimal displacement and lateral normal strain fields of a water saturated soil-pile system at a selected time instant, as evaluated using measured soil accelerations and displacements, as well as pile bending strains. Visual animation of the response of this system revealed salient complex multi-dimensional mechanisms, especially after the onset of liquefaction within the uppermost strata. Zeghal and Abdel-Ghaffar [103] used a similar technique to reconstitute the motion of Long Valley (California) earth dam. 


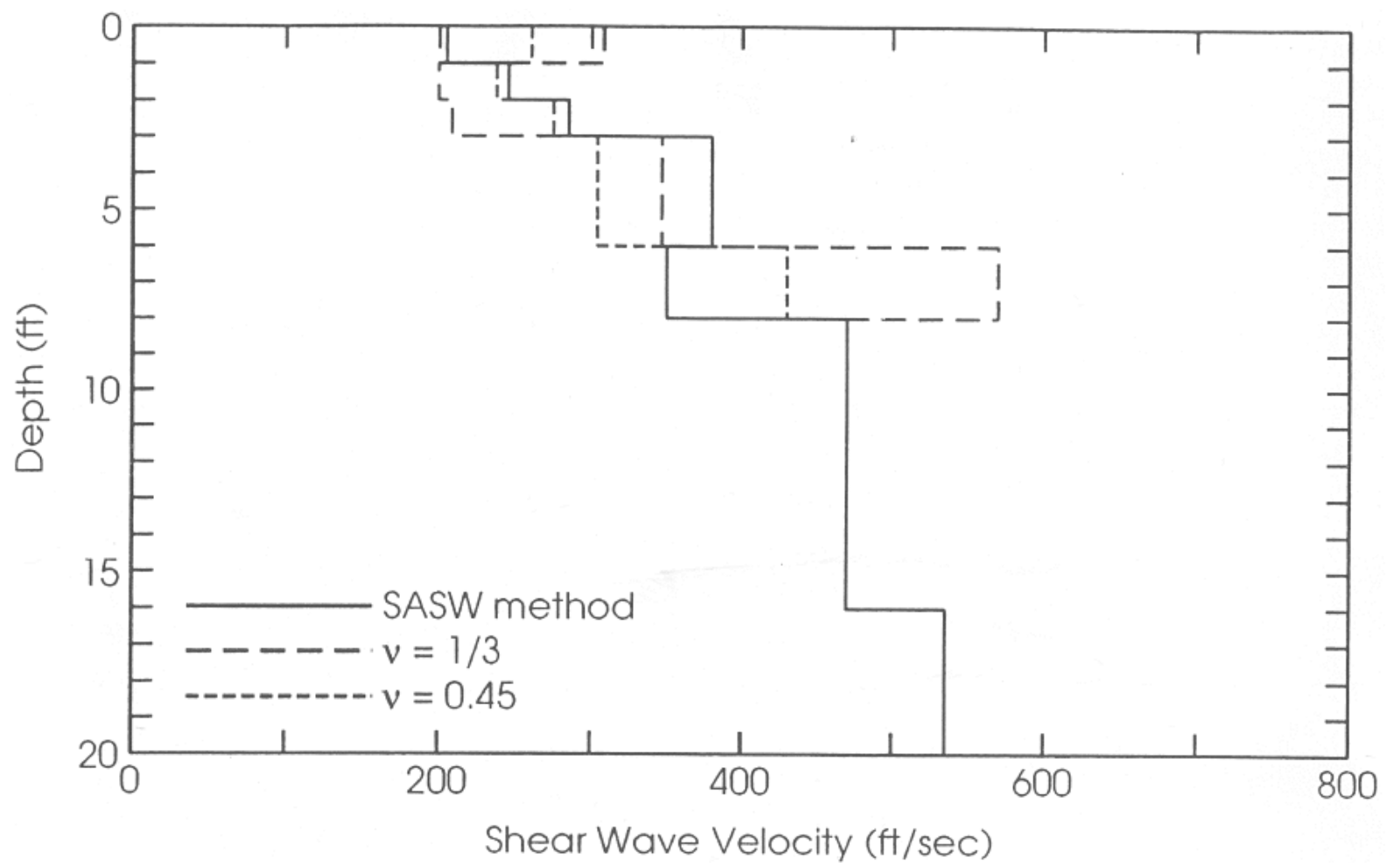

Figure 5: Shear wave velocity profile of an Imperial Valley site identified by Luco and Wong [64] using forced-vibration tests of a foundation.

\subsection{Spectral analysis of forced-vibration tests}

Non-destructive forced-vibration tests have been used to characterize the low strain in-situ dynamic properties of soil systems $[2,25]$. In this regard, spectral analysis of surface waves (SASW) method has gained wide acceptance in evaluation of site stiffness and damping parameters. SASW involves separate testing and identification phases. In testing phase, surface waves are generated by applying vertical excitations of various frequencies on the surface of a site. The generated waves are monitored using a number of motion transducers positioned on the surface away from the source of excitation. In identification phase, stiffness and possibly damping properties of underlying soils are evaluated by minimizing discrepancies between computed and experimental wave dispersion curves. SASW technique was pioneered by Stokoe and coworkers $[69,90]$ and developed further by Stokoe and others [70, 83, 101].

Forced-vibration tests of foundations were employed by Luco and Wong $[64,65]$ to identify lowstrain stiffness and damping properties of underlying soil strata. The identifications were based on a minimization of differences between theoretical impedance functions [4] and those directly computed using forced-vibration results. Figure 5 exhibits the shear wave velocities of an Imperial Valley (California) site as identified using such a foundation test data, along with velocities estimated using the SASW technique. The effects of vibration frequency range, incomplete set of impedance functions and presence of errors on the identification procedure were also studied. de Barros and Luco [22] applied a similar approach to identify impedance functions and soil properties of the surfacial layers of the Hualien (Taiwan) LSST site, and argued that the observed contrast in shear wave velocity between top and underlying layers may not be as sharp as geophysical test measurements suggested. 


\subsection{Correlation analyses}

Average seismic wave velocities of level sites were also assessed using correlation analyses of downhole acceleration records [11]. The cross-correlation function of accelerations recorded at 2 stations $i$ and $j$ (Fig. 2) reaches a major peak at a delay, $t_{d}$, which corresponds to the travel time for seismic waves to propagate between these two stations. An average velocity of wave propagation may then be computed using $v=d_{i j} / t_{d}$, in which $d_{i j}$ is the distance between stations $i$ and $j$. Elgamal, Zeghal and coworkers [29] used this approach to obtain free-field shear wave velocity profiles at the Lotung site. An averaging scheme over a number of low amplitude events was used to maximize the accuracy of estimated correlations [11]. Two major peaks were observed in the cross-correlation functions of any pair of surface and downhole accelerations, hinting the presence of incident and reflected components in recorded motions (Fig. 6). Shear wave velocity profiles of Hualien LSST site were identified using this technique by Gunturi et al. [44]. Different velocities were estimated for North-South and East-West directions, revealing evidence of stiffness anisotropy.

Wave velocities identified using cross-correlation of entire time-histories of strong-motion accelerations reflect rather an average over the range of induced strains. Correlation analyses were therefore conducted for successive time-windows which have only minor variations in motion amplitude, to assess the effect of soil softening on wave velocities. Davis [20] proposed an alternative approach to address the impact of stiffness reductions and degradations. The time step of acceleration records was compressed adaptively so that to maximize the correlation function peaks. This time compression was then used to evaluate shear wave velocity variations with time, $v=v(t)$. Specifically, wave velocities were obtained as the ratio $v(t)=d /\left(t_{d}+t_{c}(t)\right)$, in which $t_{c}(t)$ is time compression. Such estimates of wave velocities were used to identify stiffness degradation at Port Island (Japan) site during the Kobe earthquake. Cross-correlation analyses are most appropriate for coherent earthquake motions with rich frequency content. When accelerations have a narrow bandwidth, correlation functions may fail to display clearly distinct peaks. Kawakami and Haddadi [54] identified the travel velocities of incident and reflected waves between downhole array stations using a modified correlation function which improves peak separation. This function was selected to be the inverse Fourier transform of:

$$
Y\left(\omega_{i}\right)=N \Delta t\left(\frac{\frac{H\left(\omega_{i}\right)}{1+\left|H\left(\omega_{i}\right)\right|^{2}}}{\sum_{n=0}^{N-1} \frac{1}{1+\left|H\left(\omega_{n}\right)\right|^{2}}}\right), \quad i=1,2, \ldots, N
$$

in which $\omega_{i}=2 \pi i /(N \Delta t)$ is discrete frequency variable ( $i$ refers to the $i^{\text {th }}$ sample, $N$ is total number of samples, and $\Delta t$ is time step), and $H\left(\omega_{i}\right)$ is transfer function of input and output measurement pairs (note that the inverse Fourier transform of $H\left(\omega_{i}\right)$ corresponds to the usual cross-correlation function [73]). This modified cross-correlation was employed to analyze acceleration records of the Chiba, Port Island and TTRL sites in Japan, and was shown that to be effective in detecting arrival times of incident and reflected waves as well as identifying site amplification and nonlinearities (Fig. 7).

\subsection{Stress-strain imaging}

One of the pioneering investigations in geotechnical system identification using earthquake records was by Abdel-Ghaffar and Scott [1]. An identification procedure was developed to assess the dynamic 
LSST 3, 5, 6, 9, 10, 13, 14 \& 15, EW \& NS

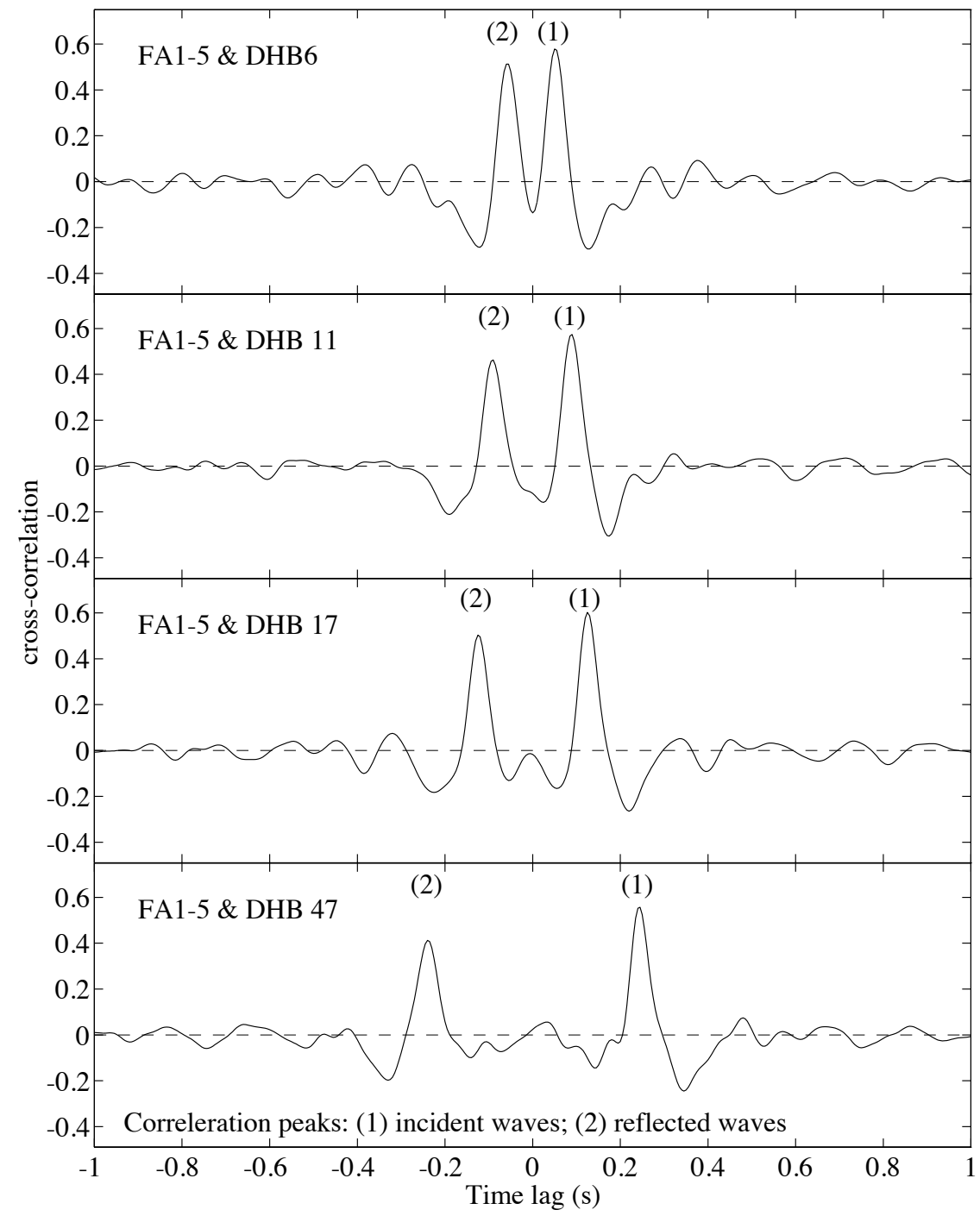

Figure 6: Cross-correlation functions of Lotung free-field accelerations (by Elgamal et al. [29]). 

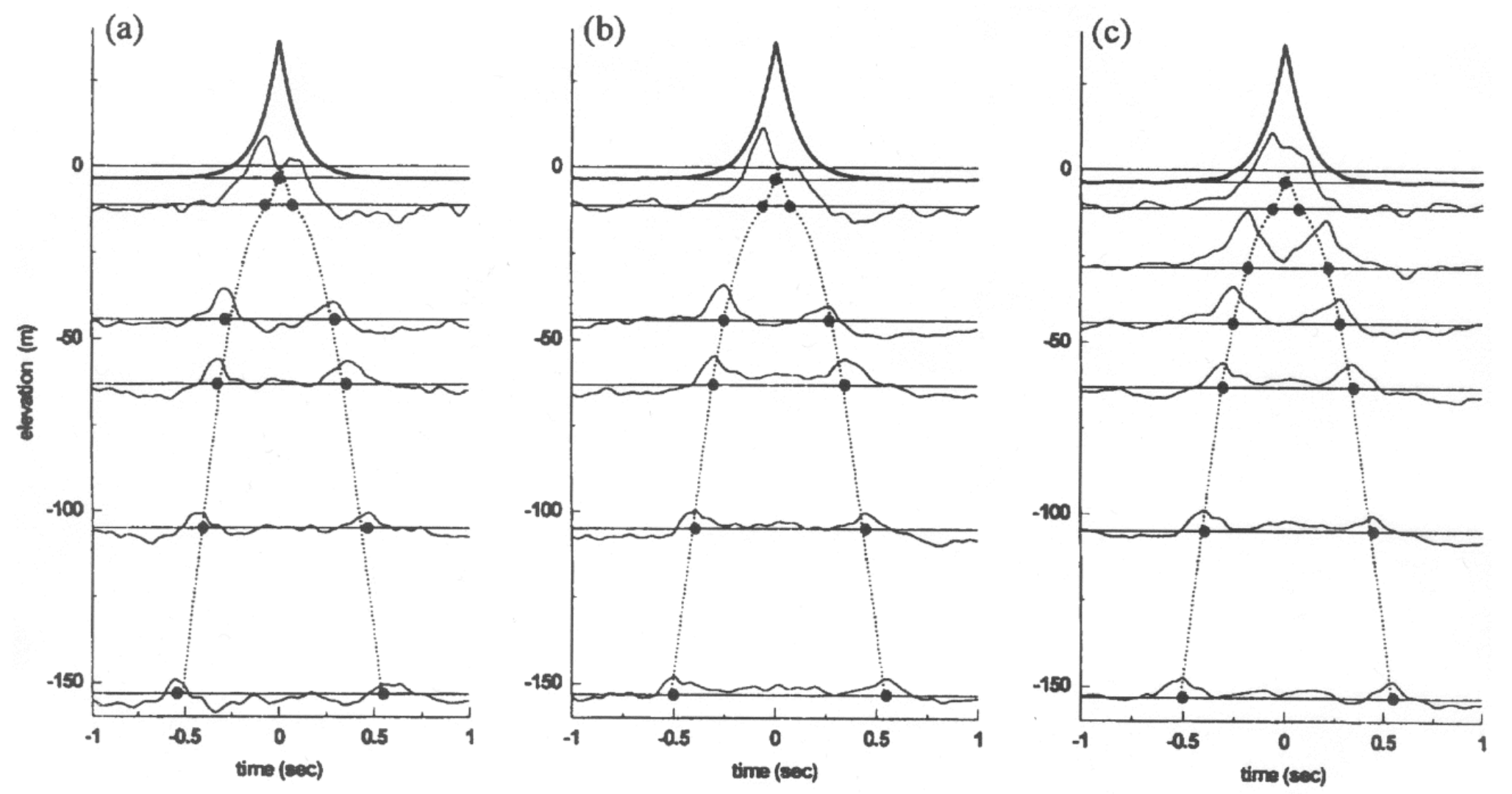

Response by NIOM

Arrival time of incident or Travel time by downhole reflectod weve by NIOM well shooting

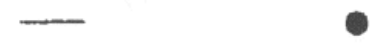

...............

Figure 7: Modified cross-correlation functions of Chiba site for three different earthquakes (as evaluated by Kawakami and Haddadi [54]). 
response of earth dams using recordings of crest and toe accelerations. The developed technique was used to analyze the response of Santa Felicia (California) earth dam during the 1976 San Fernando Earthquake. Absolute accelerations at the crest and relative displacements between the crest and toe were used, along with one- and two-dimensional shear beam models (having increasing and constant shear modulus with depth, respectively), to construct loops of the dam hysteretic response. For each hysteresis loop (Fig 8), the shear moduli for 1-D and 2-D shear beam models were then estimated using the following equations:

$$
\begin{aligned}
G_{1-\mathrm{D}} & =\rho \frac{\tilde{\Psi}(y)}{\Psi(y)}\left(\frac{v_{s o}}{\omega}\right)^{2}\left(\frac{\rho g h}{2}\right)^{1 / 3} \frac{(\ddot{x}+\ddot{z}) \max }{x_{\max }} \\
G_{2-\mathrm{D}} & =\rho\left(\frac{v_{s}}{\omega}\right)^{2} \frac{(\ddot{x}+\ddot{z}) \max }{x_{\max }}
\end{aligned}
$$

in which $v_{s o}$ and $v_{s}$ are respectively shear wave velocity parameters for the 1-D and 2-D models (as obtained using earthquake responses or field testing), $\omega$ is natural frequency of the fundamental mode of vibration evaluated using a spectral analysis, $(\ddot{x}+\ddot{z})$ max and $x$ max are respectively maximum crest acceleration and relative crest-toe displacement, $\tilde{\Psi}$ and $\Psi$ are respectively shear stress and strain modal distribution and participation factors (of the employed 1-D shear beam models), $h$ is height of dam, $\rho$ is mass density of dam material, and $g$ is gravitational acceleration. The associated damping ratios were evaluated based on estimates of energy dissipated within each hysteresis loop. Lin and Chao [60] conducted a number of nonlinear numerical simulations to assess the capabilities of the technique proposed by Abdel-Ghaffar and Scott, and suggested guidelines to obtain improved estimates of shear moduli and damping factors. The proposed approach was used to analyze earthquake recordings of Santa Felicia and Shin-San (Taiwan) dams.

Level sites and infinite slopes are statically determinate systems when subjected to 1-dimensional vertical wave propagation. Consequently, Zeghal, Elgamal, and co-workers [27, 29, 104, 107] proposed a non-parametric identification technique (referred to as stress-strain imaging) to identify shear stress-strain histories directly from downhole and surface acceleration records of such systems. Lateral shear stresses were evaluated through a simple integration of the equation of motion with respect to depth coordinate:

$$
\tau(z, t)=\int_{0}^{z} \rho \ddot{u}(\zeta, t) d \zeta
$$

in which $\tau(z, t)$ is shear stress at depth $z$ and time instant $t, \rho$ is mass density and $\ddot{u}(\zeta, t)$ is absolute lateral acceleration at level $\zeta$ and time instant $t$. Discrete estimates of these stresses were computed at the location of accelerometers and halfway in between using numerical integration techniques. The corresponding shear strains were obtained using the finite difference counterpart of:

$$
\gamma(z, t)=\frac{\partial u(z, t)}{\partial z}
$$

This technique was used to analyze the seismic response of Lotung [107], Treasure Island [29], Wildlife Refuge [107], and Port Island sites [27] as well as a number of centrifuge test models [28, 108]. The conducted analyses revealed valuable information on the mechanisms of site amplification and liquefaction, as well as on the characteristics of soil stress-strain response. Figure 9 exhibits the shear stress-strain time history of Wildlife Refuge (California) site during the 1987 Superstition Hills earthquake which was identified directly from accelerations recorded at $7.5 \mathrm{~m}$ depth and on ground 


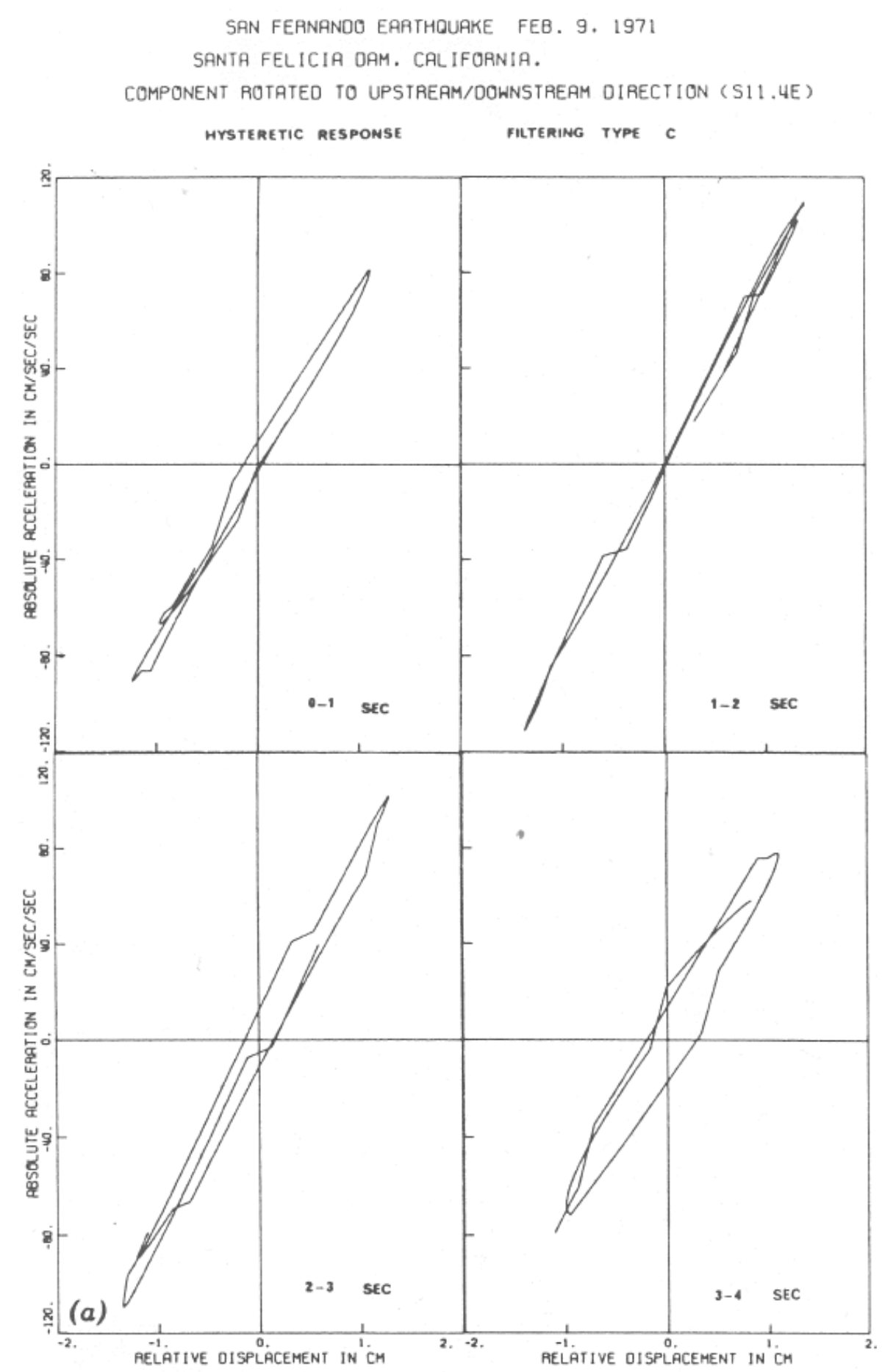

Figure 8: Acceleration-relative displacement hysteresis loops of Santa Felicia dam during San Fernando earthquake (by Abdel-Ghaffar and Scott [1]). 


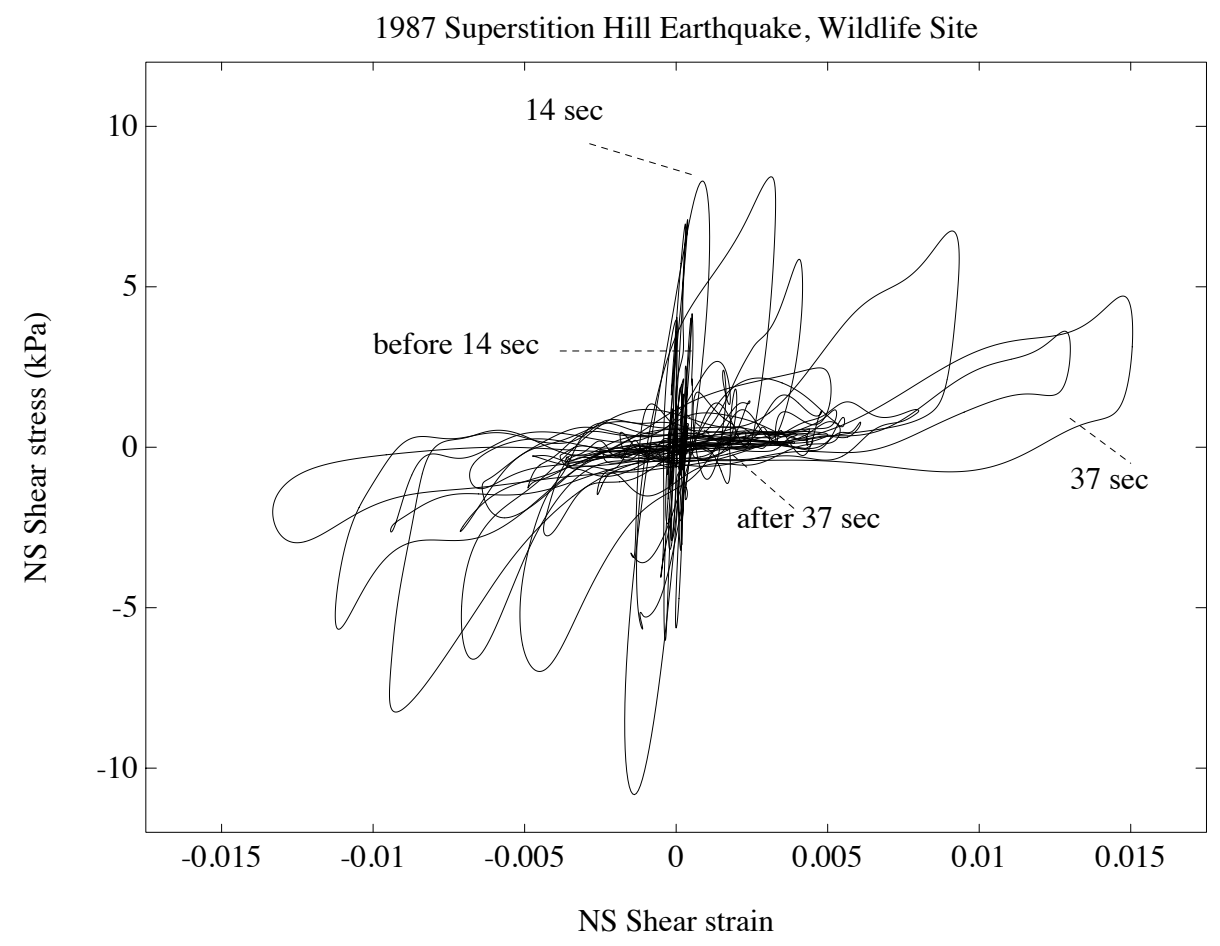

Figure 9: Shear stress-strain history of Wildlife Refuge site during the 1987 Superstition Hills earthquake( Zeghal et al. [107]).

surface. This time history revealed significant stiffness degradation associated with pore water pressure buildup, and a dilative response at large shear strains. These findings were consistent with the outcome of similar identifications conducted using experimental data of centrifuge models of level sites and infinite slopes [27, 108].

Ghayamghamian and Kawakami [38], and Taboada U. et al. [91] applied this technique to analyze earthquake acceleration records of downhole array sites in Japan, and Mexico, respectively. In a recent publication, Zeghal and Abdel-Ghaffar [103] applied the same approach to obtain approximate shear stress-strain estimates of Long Valley earth dam. Davis and Berrill [21] presented a slight modification to Zeghal and Elgamal's stress-strain imaging technique. The shear stress and strain fields were determined using a set of trigonometric interpolation functions instead of finite difference approximations. This method was applied to downhole array recordings of Port Island site.

\section{Time Series Analysis}

Realistic estimates of site specific accelerations are indispensable in seismic hazard and structural risk evaluations. Time series models have been used in simulating ground accelerations and identifying their stochastic characteristics. In these models, ground motions are idealized using random processes and calibrated by target (real) earthquake data. The calibrated models are then employed to generate synthetic accelerations having similar characteristics as target motions in terms of frequency content, variance and other characteristics.

Time series of various types have been used in ground motion simulations, including autoregressive (AR) and autoregressive-moving-average (ARMA) models, as well as non-linear Wiener time 
series [76]. Some of the ARMA models have been derived on the basis of equivalent linear idealizations of underlying physical phenomena. The time series analysis based on downhole array recordings suffer from interference effects associated with wave reflections at the ground surface as discussed for transfer function approach above (Section 2.1). This review paper presents a number of representative publications and recent studies.

\subsection{Stochastic ground motion modeling}

Stationary random processes are widely represented using the difference equation:

$$
y[t]=\sum_{i=1}^{n} a_{i} y[t-i]+\sum_{j=0}^{m} b_{j} x[t-j]
$$

in which $y[t]$ is process output at time $t$ (where $[\cdot]$ indicates a discrete process), $x[t]$ is input time series (which may be considered to be white noise), $a_{i}$ are autoregressive (AR) coefficients, $b_{i}$ are moving average (MA) coefficients, and $n$ and $m$ are AR and MA orders, respectively. When the MA order is zero $(m=0)$, Eq. 9 represents an AR model which is based solely on time-history of the measured response $y[t]$. In general, Eq. 9 represents an autoregressive moving average (ARMA) model $(m>0$ and $n>0$ ) where the effects of input history $x[t]$ are added to the AR process. Identification of such models require techniques to evaluate both AR and MA coefficients. Some of these techniques were outlined by Box and Jenkins [13].

Ground accelerations are generally non-stationary with time-varying amplitude and possibly frequency contents. Various techniques have been used to address non-stationarity in time series analysis. The simplest approach consists of dividing the acceleration records into successive pseudo-stationary pieces, and model parameters are independently identified for each piece. This approach was used by Jurkevics and Ulrych [53] to analyze the Orion Boulevard (California) acceleration records during the 1971 San Fernando earthquake (Fig. 10). An AR model was selected and the associated parameters were identified by maximizing the data entropy. Popescu and Demetriu [80] developed AR and ARMA models of a 1977 Romanian earthquake by applying a similar segmentation technique.

Earthquake accelerations may also be transformed into stationary signals through a time-dependent amplitude scaling, which is inversely proportional to the variance (i.e., modulation of the signals using an envelope function) [53]. Such an approach was used by Polhemus and Cakmak [79] to analyze the San Fernando earthquake accelerations of Orion Boulevard (Fig. 11). A polynomial function was employed to describe the variation of the acceleration variance with time, and an ARMA model was subsequently fitted to the transformed stationary series. Ólafsson and Sigbjornsson [74] used a similar envelope function approach to obtain an ARMA model for Icelandic earthquakes based on a set of 54 acceleration records. Ellis and Cakmak [32] extended this approach to the simultaneous modeling of multiple event earthquakes.

Time series models with time-varying parameters enable modeling of non-stationary ground motion, without recourse to transformations to stationary signals. Jurkevics and Ulrych [53], and Gersch and Kitagawa [36] used time varying AR models to analyze acceleration records of San Fernando earthquake. Conte et al. [19] extended this approach to ARMA models and analyzed earthquake ground motions of Lotung and El Centro (California) sites. An iterative Kalman filtering was used to evaluate the associated parameters. Skjærbæk et al. [88] compared time varying ARMA models of 1979 Imperial Valley earthquake with those based on an envelope function approach [79], and con- 


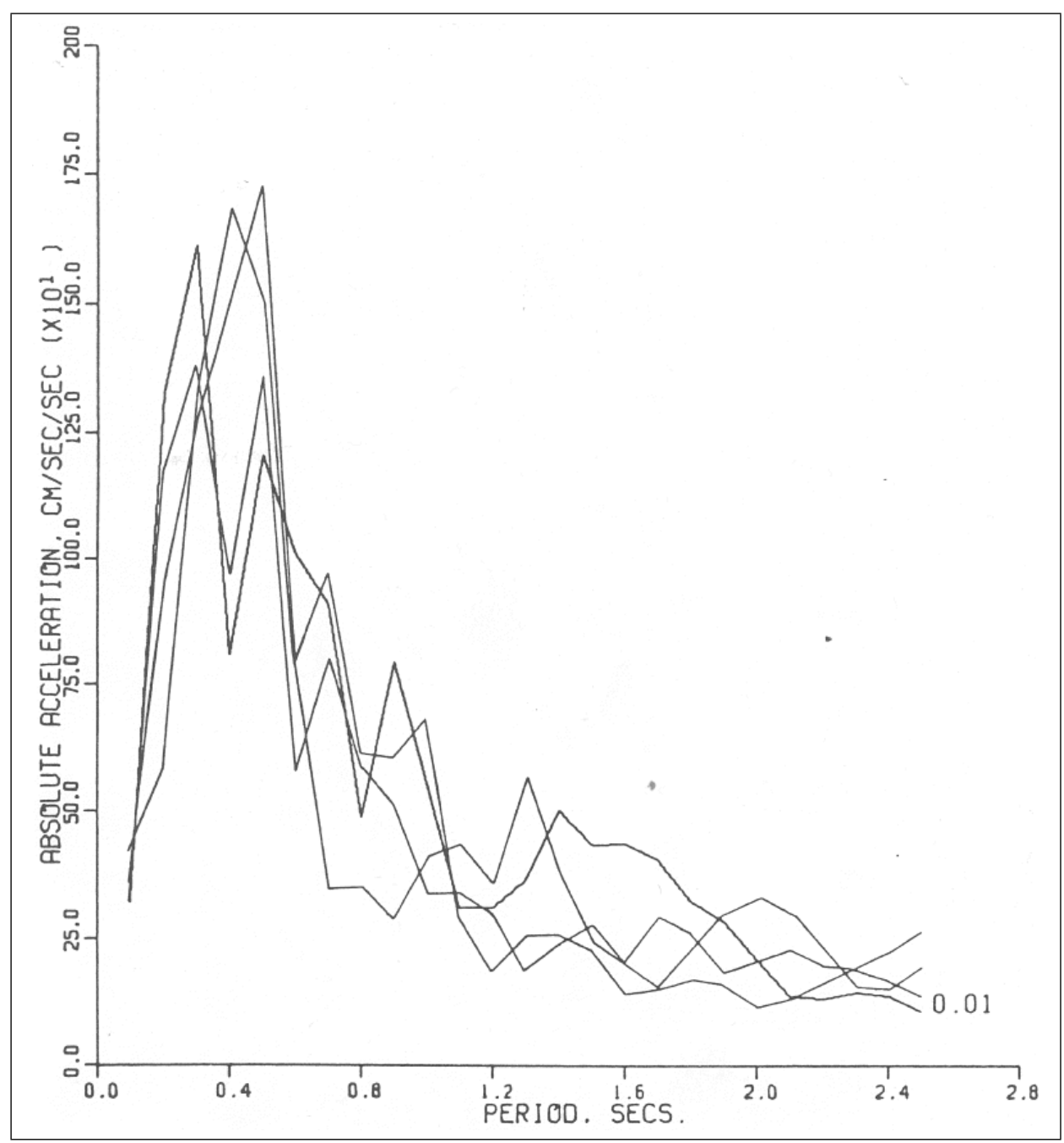

Figure 10: Acceleration response spectra of a series of simulated ground accelerations at Orion Boulevard, California (by Jurkevics and Ulrych [53]).

cluded that these techniques have comparable capabilities in addressing amplitude non-stationarity of earthquake motions.

The fundamental problem of selecting the order of time series models was partially addressed by Chang et al. [18]. Time series of orders 2,1 (i.e., $n=2$ and $m=1$ ) and 4,1 were argued to be effective in modeling California ground accelerations. This study also concluded that the time-dependent variance was the main contributor to non-stationarity of these accelerations.

\subsection{Time series modeling using downhole array records}

Surface and downhole accelerations of sites may be used as input-output pairs in time series models ( $x$ and $y$ respectively, in Eq. 9). Glaser [41] analyzed site amplification and liquefaction at Wildlife Refuge site during Elmore Ranch and Superstition Hills earthquakes using surface and $7.5 \mathrm{~m}$ depth accelerations records. The site was idealized using a multi-degree-of-freedom system and modeled using time-dependent ARMA models. The associated parameters were estimated using a recursive Kalman filter algorithm, and linked to modal frequencies, critical damping and power participation 

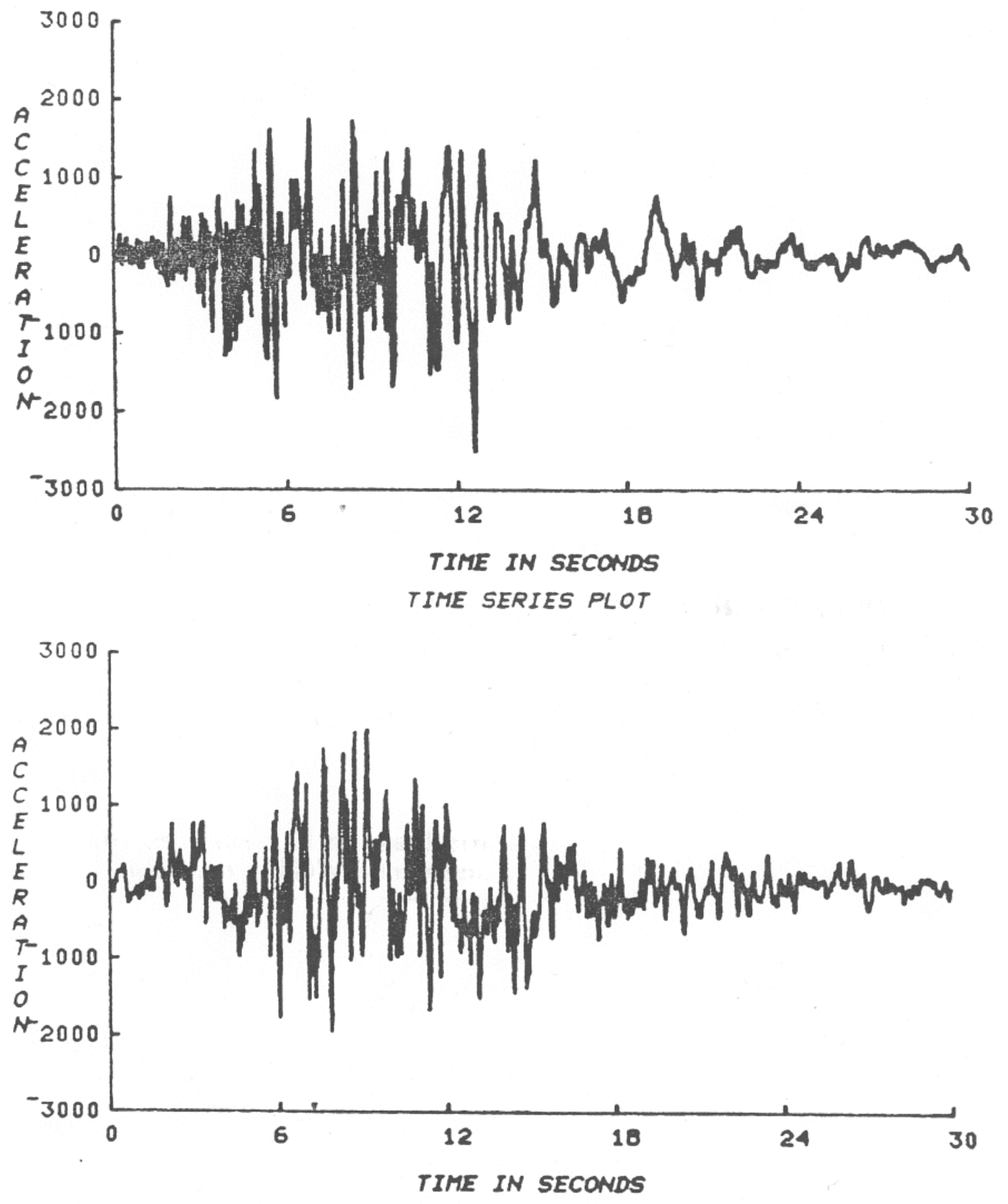

Figure 11: Recorded acceleration history (top) at Orion Boulevard during the San Fernando earthquake and corresponding time series simulation (bottom, as evaluated by Polhemus and Cakmak [79]). 


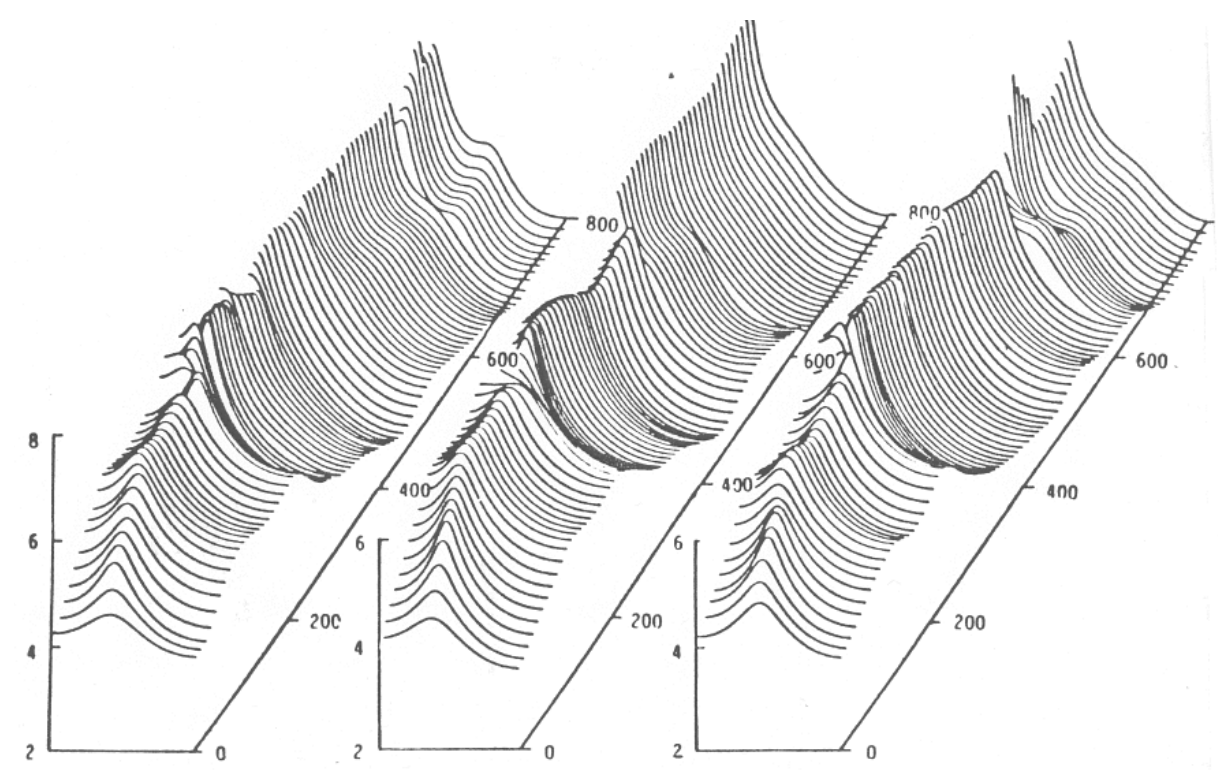

Figure 12: Instantaneous spectrum analysis for San Fernando earthquake and two simulated accelerograms [36].

factors (Fig. 13). Baise, Glaser and co-workers [6, 7] utilized time dependent ARMA models to assess the impact of soil nonlinearities on site response at Chiba, Garner Valley (California), Lotung, and Port Island. Consistency of the estimated models was investigated by predicting site response to recorded earthquakes other than the ones used in identification of time series parameters.

\section{Identifications Involving Constitutive Modeling}

Constitutive soil models have reached high levels of refinement and sophistication [113] in view of drastic improvements in computational tools and testing technologies over the last decades. The predictive capabilities of these models remain questionable as long as they have not been calibrated using real earthquake or other strong-motion excitations of full-scale soil systems. However, only limited system identification research has be undertaken in this regard. Non-parametric as well as parametric models have been used in identification analyses of soil constitutive models.

\subsection{Nonparametric modeling}

Non-parametric models are commonly black-box representations aimed at idealizing system behavior based on observed response and possibly input excitations. Some non-parametric models have infinite dimensions and introduce only minor constraints to the input-output relationship. Such non-parametric models are generally capable of reproducing experimental or observational response data with a good quality of fitness. However, the internal structure of these models is commonly algorithmic and may not reflect the involved physical phenomena.

Artificial neural networks have been used by a number of researchers to idealize soil stress-strain relationships. Ellis et al. [33] used a sequential neural network to model the constitutive behavior of sandy materials. This model was identified using a series of triaxial compression experiments, and has the ability to simulate unload-reload cycles and accounts for the impact of grain size distributions. 


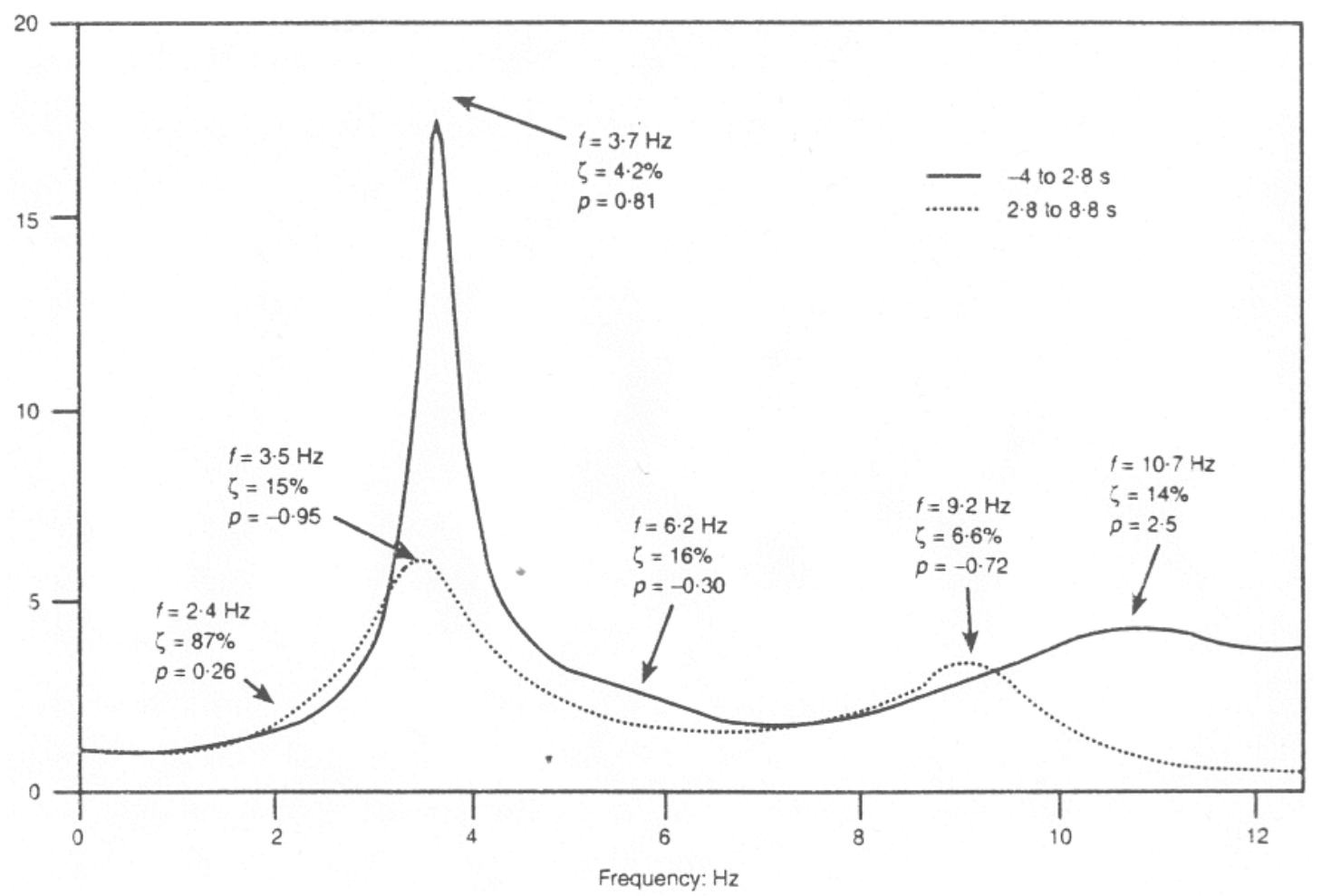

Figure 13: Stationary spectral estimates with computed natural frequency, damping ratio and participation factor for Wildlife site during Superstition Hills earthquake [41].

Sidarta and Ghaboussi [87] modeled the soil constitutive behavior when subjected to non-uniform stress conditions. The proposed neural network model was calibrated using finite element simulations of triaxial compression tests. The calibrated model was thereafter used to predict soil response to a series of actual triaxial experiments with end friction (non-uniform stress conditions). Zhu et al. [112] developed a recursive neural network model to predict shear behavior of granular soils. This model is capable of simulating the dilation and contraction of dense and loose soil samples, respectively. Basheer, Najjar, and coworkers $[8,68]$ used static and recurrent artificial neural networks to model the constitutive response of fine grained soils to cyclic loads and argued that recurrent artificial neural networks were more effective than static ones. Goh $[42,43]$ investigated the capabilities of artificial neural networks in the assessment of site liquefaction potential based on cone penetration test (CPT) data. A series of CPT measurements from worldwide sites were employed to train and test artificial neural network models, which successfully predicted the liquefaction response.

\subsection{Parametric modeling}

Parametric models commonly present phenomenological descriptions of the involved response mechanisms. The main advantage of employing parametric models is that the associated parameters often have physical significance and characterize properties of the physical system (e.g. shear modulus as a function of strain amplitude, $G=G(\gamma)$, damping, and others). The objective of the parametric model identification is to calibrate a selected model by estimating a set of parameters that provides an optimal match between recorded and computed responses. The estimated parameters commonly include moduli and damping factors, inelastic state variables, among others. When properly calibrated, a para- 
metric constitutive model is often expected to predict the soil response over a range of conditions. The number of parameters that can be identified and the reliability of the identified parameters is directly related to the availability of experimental data over the range of response domain of interest. Identification studies based on insufficient experimental data may lead to inaccuracies in the identified model parameters, as well as ill-posedness of the inverse problem as further discussed below. A number of identification techniques were used by researchers to calibrate parametric soil models, ranging from simple qualitative comparisons to sophisticated Bayesian approaches (e.g., [31, 95, 102]).

\subsubsection{Statically determinate systems}

The identification problem of distributed parameter systems commonly involves solution of the equations of motion and constitutive relations. The problem may be simplified substantially when a system is statically determinate. The equations of motion of these systems suffice to directly identify the associated stresses and strains from acceleration records (as discussed above for level sites). Such stress-strain estimates may then be employed as input-output (strain-stress) pairs in constitutive model calibrations.

Elgamal, Zeghal and coworkers identified the evolution of stiffness and damping parameters of a number of level sites [29, 106] and landfills [26] by minimizing the discrepancies between stress histories obtained through stress-strain imaging technique, and those predicted by equivalent linear visco-elastic models. Successive overlapping short time windows were used to account for nonlinearities. This method was used to study the impact of site amplification and liquefaction on soil stiffness properties. Later, Gunturi et al. [44] applied this technique to analyze the Hualien site. This study revealed a significant anisotropy in the soil stiffness properties, in agreement with the correlation analyses mentioned above. In a recent publication, Carvajal et. al. [16] analyzed earthquake recordings from Central de Abasto Oficinas (Mexico) downhole array with linear visco-elastic models. The effects of frequency contents of earthquake excitations on identified stiffness and viscous damping parameters were investigated.

The dynamic response of statically determinate systems may also be identified using a boundary value problem formulation restricted to the zone equipped with accelerometers. Such sub-system analyses are possible since a limited number of motion records is sufficient to fully define the boundary conditions (of the sub-system). For level sites equipped with downhole arrays, identification analyses may be therefore restricted to instrumented upper soil strata. The boundary input motion required for such analyses is provided by measured accelerations at the base of these strata. Loh and Yeh [63] analyzed the seismic response of the top $47 \mathrm{~m}$ layer of Lotung site using an equivalent linear soil constitutive model. The stiffness and damping coefficients were obtained for successive 4-second time intervals using a recursive least squares method. Loh and Yeh [63] also used a bilinear hysteretic model along with an extended Kalman filter to analyze soil nonlinear response at Lotung. This bilinear model was found to be more effective than an equivalent linear one in idealization of soil behavior at this site.

\subsubsection{Bayesian Approach}

Soil-structures and natural deposits are generally massive systems which have distributed parameters and state. Earthquake records of such systems commonly do not provide sufficient information to fully develop a model of the system response and identify a unique set of model parameters. Bayesian identification techniques have been used to mathematically regularize the problem and eliminate or partially reduce its indeterminacy. 
Udwadia et al. [95] investigated the determinacy of parameter identification problems in structural and geotechnical engineering. Two different types of ill-posedness were defined. An identification problem is inherently ill-posed when the employed experimental data (of response and potentially input measurements) yields non-unique sets of parameters. The problem is said to be algorithmically ill-posed when parameter identification is unstable in the presence of measurement noise. A Bayesian technique was proposed to tackle the identification of spacially varying system stiffness parameters when measurements are corrupted by Gaussian random noise.

Zeghal and Abdelghaffar [102] also used a Bayesian technique to identify and analyze the nonlinear seismic response of Long Valley earth dam. The problem was formulated as a combination of a priori and experimental information with theoretical knowledge. An elastoplastic constitutive relationship along with a Von-Mises yield criterion were used to model the clayey soil of Long Valley earth dam [81]:

$$
d \boldsymbol{\sigma}=\left(\mathbf{E}-\frac{(\mathbf{E}: \mathbf{Q})(\mathbf{Q}: \mathbf{E})}{H_{p}-H_{o}}\right): d \boldsymbol{\varepsilon}
$$

in which $\boldsymbol{\sigma}$ and $\boldsymbol{\varepsilon}$ are respectively total stress and strain tensors, $\mathbf{E}$ is elastic constitutive tensor (as a function of the soil elastic shear modulus, $G_{o}$ and Poisson's ratio $\left.v\right), \mathbf{Q}$ is unit outward normal to the yield surface, $H_{o}$ and $H_{p}$ are respectively elastic and plastic parameters (for a Von-Mises criterion, $H_{p}=1 /\left(1 / 2 G-1 / 2 G_{o}\right)$ where, $G$ is tangent shear modulus and $\left.H_{o}=2 G_{o}\right)$. The identification analyses were restricted to shear modulus variation with strain amplitude, which was recognized as the most influential model parameter affecting the dam response. The conducted analyses of Long Valley earth dam revealed a complex 3-dimensional response that was marked by a shear stress-strain behavior slightly more elastic than trends observed in triaxial tests. Sayed and Abdel-Ghaffar [85] applied a similar technique to earthquake records of Anderson and Puddingstone (California) earth dams. Honjo and Kudo [47] used an alternative approach in combining observational data with a-priori information [72]. Soil stiffness parameters of an embankment in Yamagata (Japan) were identified using deformation and pore-pressure recordings (as observed during construction) by maximization of the relative entropy.

Lin $[58,59]$ used an extended Kalman filter to analyze the Lotung site using the LSST downhole array records. This filter is an unbiased, minimum variance, and sequential estimation scheme which incorporates available information through a priori estimates of the mean and the covariance matrix of state and parameter vectors. A Bouc-Wen model was employed to idealize the lateral shear stressstrain relationship:

$$
\dot{\tau}=G \dot{\gamma}-\alpha|\dot{\gamma}||\tau|^{n-1} \tau-\beta \dot{\gamma}|\tau|^{n}
$$

in which $\tau$ and $\gamma$ are respectively shear stress and strain, $G$ is initial (low-strain) shear modulus, and $\alpha, \beta$ and $n$ are model parameters. The hysteretic behavior of soils was addressed using generalized Masing's rule. Figure 14 displays identified shear modulus reduction and damping curves along with results of laboratory soil sample tests.

\subsubsection{Local Identification}

Identifying and calibrating models of the dynamic constitutive response of a full-scale soil system is a challenging task, especially when this response is monitored using a limited number of sensors. The problem is complicated further when the system boundary conditions are not well defined (e.g., semi infinite sites). Zeghal and Oskay [75, 110] developed a novel local identification technique that uses the acceleration and pore-pressure records provided by dense arrays of accelerometers and pore 

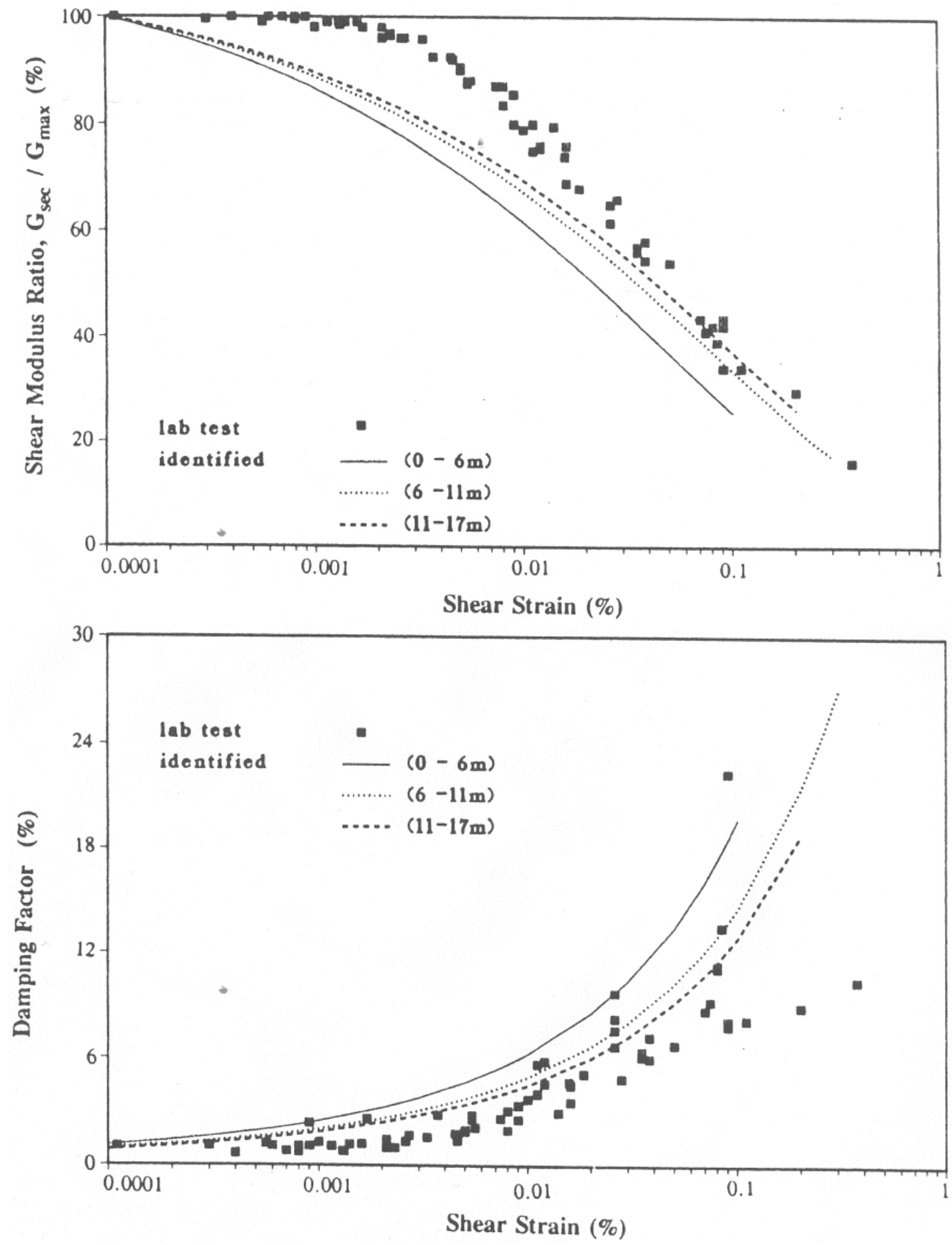

Figure 14: Comparison of identified and measured shear modulus ratios and damping factors [59]. 


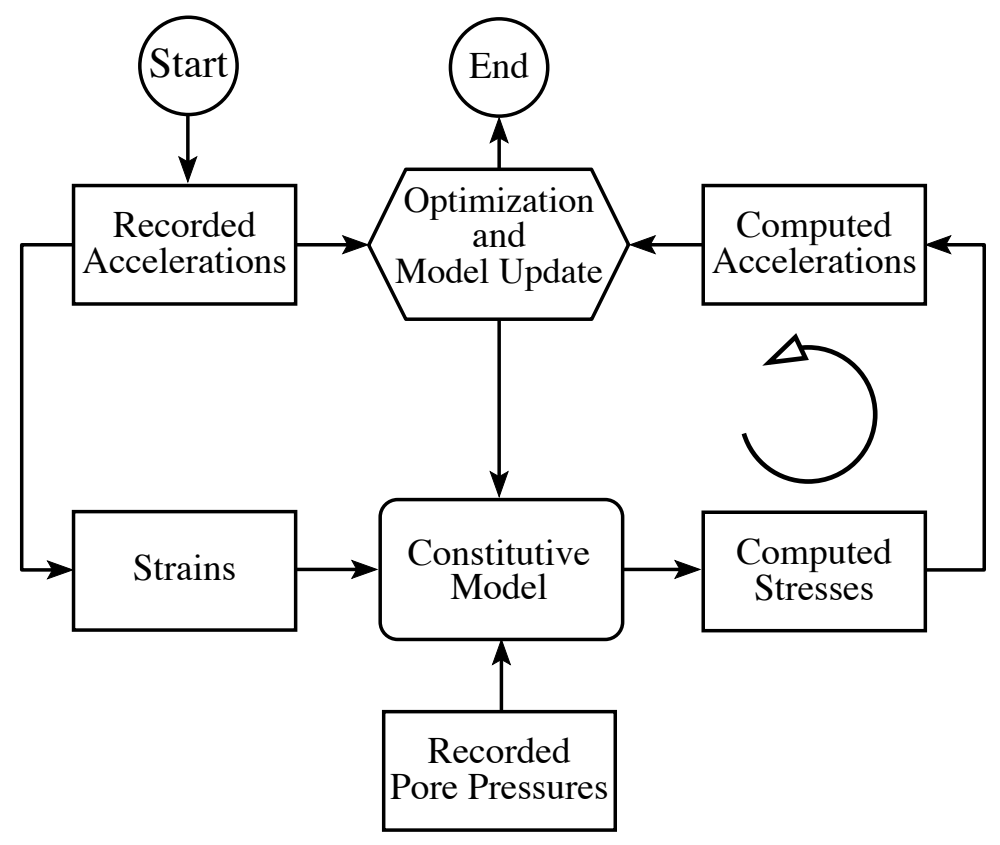

Figure 15: Algorithm for local system identifications (Zeghal and Oskay [111]).

pressure transducers (Fig. 15). This approach reduces the indeterminacy of the problem by limiting the identifications to a local zone. The developed technique does not require the availability of boundary condition measurements, or solution of a boundary-value-problem associated with the observed system. A multi-surface plasticity was used to model the hysteretic and path dependent stress-strain response of soils. The identification algorithm was benchmarked using numerical simulations and centrifuge models (Fig. 16) of 2-D quay wall-soil systems [110]. The concept of employing experimental data sets and local in-situ measurements to calibrate complex constitutive laws with large number of parameters have been systematically investigated by Yang and Elgamal [100], Levasseur et al. [56, 57], Hashash et al. [46, 35], and Calvello and Finno:[15].

\section{Conclusions}

This paper presented a survey of identification techniques and analyses of geotechnical systems with emphasis on earthquake engineering applications. The surveyed studies were subdivided into 3 categories: (1) response characterization and pattern recognition studies, (2) time series analyses, and (3) constitutive (stress-strain) model identifications. Pattern recognition and non-parametric identifications, such as spectral analysis, stress-strain imaging and cross-correlation analyses provide valuable insight into the system properties and response mechanisms without recourse to explicit modeling of the involved physical phenomena. Time series provide tools to model the stochastic process of ground motion accelerations, and have been used to generate synthetic accelerations having similar characteristics as those of target earthquakes. Non-parametric as well as parametric models have been used in identification analyses of constitutive response of soil systems. Bayesian and other techniques were employed to reduce the identification problem indeterminacy. In the near future, system identification analyses are destined to play an ever important role in assessment and modeling of geotechnical systems in view of continuous drastic improvements in sensor and testing technologies. 

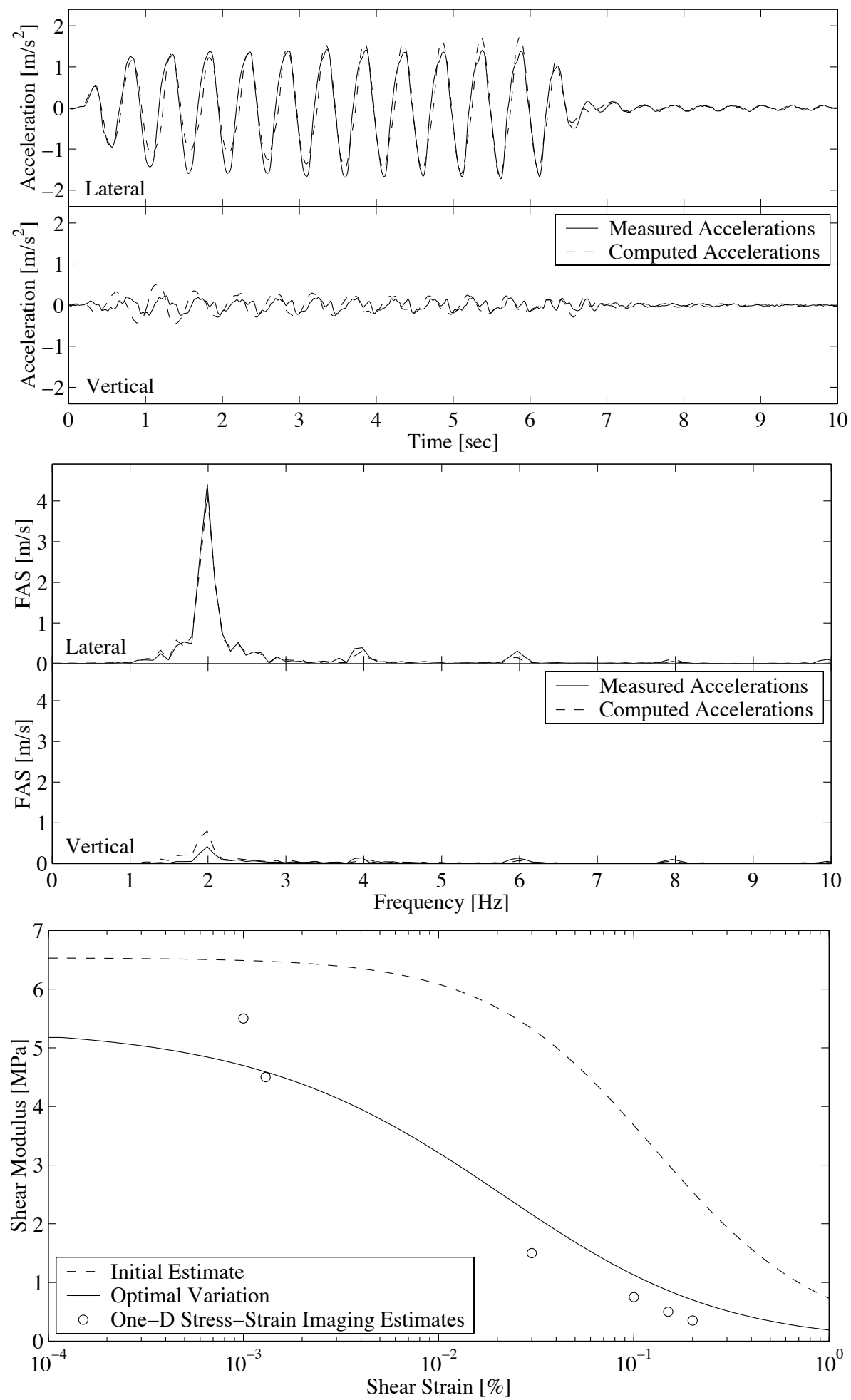

Figure 16: Local identification of a soil-quay wall centrifuge model subjected to a strong shaking: acceleration time histories, Fourier amplitude spectra, and shear moduli at the central instrument station (by Zeghal and Oskay [110]). 


\section{Acknowledgements}

This research was supported by the National Science Foundation, Grant No. CMS-9984754. This support is gratefully acknowledged.

\section{References}

[1] Abdel-Ghaffar, A. M. and Scott, R. F. (1979). "Shear moduli and damping factors of earth dam." Journal of the Geotechnical Engineering Division, ASCE, 105(GT12), 1405-1426.

[2] Abdel-Ghaffar, A. M. and Scott, R. F. (1981). "Vibration tests of full-scale earth dam." Journal of the Geotechnical Engineering Division, ASCE, 107(GT3), 241-269.

[3] Aguirre, J. and Irikura, K. (1997). "Nonlinearity, liquefaction, and velocity variation of soft soil layers in port island, kobe, during the hyogo-ken nanbu earthquake." Bulletin of Seismological Society of America, 87(5), 1244-1258.

[4] Apsel, R. J. and Luco, J. E. (1987). "Impedance functions for foundations embedded in a layered medium, an integral equation approach.” Earthquake Eng. and Struct. Dyn., 15(2), 213-231.

[5] Astrom, K. J. and Eykhoff, P. (1971). "System identification - a survey.” Automatica, 7, 123-162.

[6] Baise, L. G. and Glaser, S. D. (2000). "Consistency of ground-motion estimates made using system identification.” Bulletin of the Seismological Society of America, 90(4), 993-1009.

[7] Baise, L. G., Glaser, S. D., and Sugano, T. (2001). "Consistency of dynamic site response at port island.” Earthquake Engng Struct. Dyn., 30, 803-818.

[8] Basheer, I. A. and Najjar, Y. M. (1998). "Modeling cyclic constitutive behavior by neural networks: Theoretical and real data." Proc. of the 12th Engineering Mechanics Conference, $\mathrm{H} . \mathrm{Mu}-$ rakami and J. E. Luco, eds., ASCE, La Jolla, California. 952-955.

[9] Beck, J. L. (1996). "System identification methods applied to measured seismic response.” Proceedings of the Eleventh World Conference on Earthquake Engineering, number 2004, Acapulco, Mexico. Elsevier.

[10] Bendat, J. S. (1990). Nonlinear System Analysis and Identification from Random Data. John Wiley and Sons, New York, New York.

[11] Bendat, J. S. and Piersol, A. G. (1980). Engineering Applications of Correlation and Spectral Analysis. John Wiley and Sons, New York, New York.

[12] Beresnev, A. B., Wen, K.-L., and Yeh, Y. T. (1995). "Seismological evidence for nonlinear elastic ground behavior during large earthquakes." Soil Dynamics and Earthquake Engineering, 14, 103-114.

[13] Box, G. E. P. and Jenkins, G. M. (1976). Time Series Analysis: Forecasting and Control. HoldenDay, San Francisco, California. 
[14] Bui, H. D. (1993). Inverse Problem in the Mechanics of Materials: An Introduction. CRC Press, Boca Raton, Florida.

[15] Calvello, M. and Finno, R. J. (2004). "Selecting parameters to optimize in model calibration by inverse analysis." Computers and Geotechnics, 31, 411-425.

[16] Carvajal, U. J. C., Taboada, U. V. M., and Romo, O. M. P. (2002). "Influence of earthquake frequency content on soil dynamic properties at cao site." Soil Dynamics and Earthquake Engineering, 22, 297-308.

[17] Chang, C. Y., Mok, C. M., and Tang, H. T. (1996). "Inference of dynamic shear modulus from lotung downhole data." Journal of Geotechnical Engineering, ASCE, 122(8), 657-665.

[18] Chang, M. K., Kwiatkowski, J. W., Nau, R. F., Oliver, R. M., and Pister, K. S. (1982). "Arma models for earthquake ground motions." Earthquake Engng Struct. Dyn., 10, 651-662.

[19] Conte, J. P., Pister, K. S., and Mahin, S. A. (1992). "Nonstationary arma modeling of seismic motions." Soil Dynamics and Earthquake Engineering, 11, 411-426.

[20] Davis, R. O. (2000). "Estimation of soil shear modulus softening during strong ground shaking using ground surface and downhole acceleration recordings." Earthquake Engng Struct. Dyn., 29, 359-376.

[21] Davis, R. O. and Berrill, J. B. (1998). "Rational approximation of stress and strain based on downhole acceleration measurements." International Journal for Numerical and Analytical Methods in Geomechanics, 22, 603-619.

[22] de Barros, F. C. P. and Luco, J. E. (1995). "Identification of foundation impedance functions and soil properties from vibration tests of the hualien containment model." Soil Dynamics and Earthquake Engineering, 14, 229-248.

[23] Elgamal, A., Lai, T., Wilson, D., and Kutter, B. (2001). "Identification of small strain dynamic properties of dense sand." Proceedings: Fourth International Conference on Recent Advances in Geotechnical Earthquake Engineering and Soil Dynamics and Symposium in Honor of Professor W. D. Liam Finn, S. Prakash, ed., number 1.67, San Diego, California.

[24] Elgamal, A., Lai, T., Yang, Z., and He, L. (2001). "Dynamic soil properties, seismic downhole arrays and applications in practice." Proceedings: Fourth International Conference on Recent Advances in Geotechnical Earthquake Engineering and Soil Dynamics and Symposium in Honor of Professor W. D. Liam Finn, S. Prakash, ed., number SOAP-6, San Diego, California. State-of-theart paper.

[25] Elgamal, A. W., Alampalli, S., and Van Laak, P. (1996). "Forced vibration of full-scale wallbackfill system." Journal of Geotechnical Engineering, ASCE, 122(10), 849-858.

[26] Elgamal, A. W., Lai, T., Gunturi, R., and Zeghal, M. (2004). "System identification of landfill seismic response." Journal of Earthquake Engineering, 8, 545-566.

[27] Elgamal, A. W., Zeghal, M., Dobry, R., and Taboada, V. M. (1996). "Analysis of site liquefaction and lateral spreading using centrifuge testing records.” Soils and Foundations, 36(2), 111-121. 
[28] Elgamal, A. W., Zeghal, M., and Parra, E. (1996). "Liquefaction of reclaimed island in kobe, japan.” Journal of Geotechnical Engineering, ASCE, 122(1), 39-49.

[29] Elgamal, A. W., Zeghal, M., Parra, E., Gunturi, R., Tang, H. T., and Stepp, J. C. (1996). "Identification and modeling of earthquake ground response i: Site amplification." Soil Dynamics and Earthquake Engineering, 15, 499-522.

[30] Elgamal, A. W., Zeghal, M., Tang, H. T., and Stepp, J. C. (1995). "Lotung downhole array. i: Evaluation of site dynamic properties." Journal of Geotechnical Engineering, ASCE, 121(4), 350362.

[31] Elgamal, A. W. M., Scott, R. F., F., S. M., and L., Y. (1990). "La villita dam response during five earthquakes including permanent deformation." Journal of Geotechnical Engineering, ASCE, 116(10), 1443-1462.

[32] Ellis, G. W. and Cakmak, A. S. (1991). "Time series modelling of strong ground motion from multiple event earthquakes." Soil Dynamics and Earthquake Engineering, 10(1), 42-53.

[33] Ellis, G. W., Yao, C., Zhao, R., and Penumadu, D. (1995). "Stress-strain modeling of sands using artificial neural networks.” Journal of Geotechnical Engineering, ASCE, 121(5), 429-435.

[34] Eykhoff, P. (1974). System Identification. John Wiley and Sons, Toronto, Canada.

[35] Fu, Q., Hashash, Y. M. A., Jung, S., and Ghaboussi, J. (2007). "Integration of laboratory testing and constitutive modeling of soils." Computers and Geotechnics, 34, 330-345.

[36] Gersch, W. and Kitagawa, G. (1985). "A time varying ar coefficient model for modelling and simulating earthquake ground motion.” Earthquake Engng Struct. Dyn., 13, 243-254.

[37] Ghanem, R. and Shinozuka, M. (1995). "Structural system identification i: Theory." Journal of Engineering Mechanics, ASCE, 121(2), 255-264.

[38] Ghayamghamian, M. R. and Kawakami, H. (2000). "On-site nonlinear hysteresis curves and dynamic soil properties." Journal of Geotechnical and Geoenvironmental Engineering, ASCE, 126(6), $543-555$.

[39] Ghayamghamian, M. R. and Motosaka, M. (2001). "Identification of dynamic soil properties using vertical array recordings." Proceedings: Fourth International Conference on Recent Advances in Geotechnical Earthquake Engineering and Soil Dynamics and Symposium in Honor of Professor W. D. Liam Finn, S. Prakash, ed., number 3.33, San Diego, California.

[40] Glaser, S. D. (1995). "System identification and its application to estimating soil properties." Journal of Geotechnical Engineering, ASCE, 121(7), 553-560.

[41] Glaser, S. D. (1996). "Insight into liquefaction by system identification." Géotechnique, 46(4), 641-655.

[42] Goh, A. T. C. (1996). "Neural-network modeling of cpt seismic liquefaction data." Journal of Geotechnical Engineering, ASCE, 122(1), 70-73. 
[43] Goh, A. T. C. (2002). "Probabilistic neural network for evaluating seismic liquefaction potential." Canadian Geotechnical Journal, 39(1), 219-232.

[44] Gunturi, V. R., Elgamal, A. W. M., and Tang, H. T. (1998). "Hualien seismic downhole data analysis." Engineering Geology, 50, 9-29.

[45] Hart, G. and Yao, J. (1977). "System identification in structural dynamics." Journal of Engineering Mechanics Division, ASCE, 103(EM6), 1089-1104.

[46] Hashash, Y. M. A., Marulanda, C., Ghaboussi, J., and Jung, S. (2006). "Novel approach to integration of numerical modeling and field observations for deep excavations." Journal of Geotechnical and Geoenvironmental Engineering, ASCE, 132(1019-1031).

[47] Honjo, Y. and Kudo, N. (1998). "Matching objective and subjective information in geotechnical inverse analysis based on entropy minimization." Inverse Problems in Engineering Mechanics, M. Tanaka and G. Dulikravich, eds., Nagano City, Japan. Elsevier, 263-271.

[48] Hoshiya, M. and Maruyama, O. (1987). "Identification of nonlinear structural systems." Proceedings of ICASP-5, Vol. 1, Vancouver, Canada. 182-189.

[49] Huang, H.-C., Shieh, C.-S., and Chiu, H.-C. (2001). "Linear and nonlinear behaviors of soft soil layers using lotung downhole array in taiwan." TAO, 12(3), 503-524.

[50] Imai, H., Yun, C.-B., Maruyama, O., and Shinozuka, M. (1989). "Fundamentals of system identification in structural dynamics." Probabilistic Engineering Mechanics, 4(4), 162-173.

[51] Johansson, R. (1993). System Identification and Modeling. Prentice Hall, Englewood Cliffs, New Jersey.

[52] Juang, J.-N. (1993). Applied System Identification. Prentice Hall, Englewood Cliffs, New Jersey.

[53] Jurkevics, A. and Ulrych, T. J. (1978). "Representing and simulating strong ground motion." Bulletin of the Seismological Society of America, 68(3), 781-801.

[54] Kawakami, H. and Haddadi, H. R. (1998). "Modeling wave propagation by using normalized input-output minimization (niom)." Soil Dynamics and Earthquake Engineering, 17, 117-126.

[55] Kozin, F. and Natke, H. (1986). "System identification techniques.” Structural Safety, 3, 269316.

[56] Levasseur, S., Malecot, Y., Boulon, M., and Flavigny, E. (2009). "Statistical inverse analysis based on genetic algorithm and principal component analysis: Method and developments using synthetic data." International Journal for Numerical and Analytical Methods in Geomechanics, 33(12), 1485-1511.

[57] Levasseur, S., Malecot, Y., Boulon, M., and Flavigny, E. (2010). "Statistical inverse analysis based on genetic algorithm and principal component analysis: Applications to excavation problems and pressuremeter tests." International Journal for Numerical and Analytical Methods in Geomechanics, 34(5), 471-491. 
[58] Lin, J.-S. (1990). "Nonlinear system identification of soils using extended kalman filter." Proceedings of Fourth U.S. National Conference of Earthquake Engineering, Vol. 3, EERI, Palm Springs, California. 865-874.

[59] Lin, J.-S. (1994). "Extraction of dynamic soil properties using extended kalman filter." Journal of Geotechnical Engineering, ASCE, 120(12), 2100-2117.

[60] Lin, J.-S. and Chao, B.-K. (1990). "Estimation of shear moduli and damping factors of earth dam materials." Earthquake Engng Struct. Dyn., 19, 891-910.

[61] Ljung, L. J. (1999). System Identification: Theory for the User. Prentice Hall, 2 edition.

[62] Ljung, L. J. and Glover, K. (1981). "Frequency domain versus time domain methods in system identification." Automatica, 17(1), 71-86.

[63] Loh, C. H. and Yeh, C. S. (1992). "Identification of site response using 3-d array records." Proceedings of the 10th Conference on Earthquake Engineering, Vol. 1, Madrid, Spain. A. A. Balkema, 259-263.

[64] Luco, J. E. and Wong, H. L. (1990). "Identification of soil properties from vibration tests of small footings." Proceedings of Fourth U.S. National Conference on Earthquake Engineering, Vol. 3, EERI, Palm Springs, California. 875-884.

[65] Luco, J. E. and Wong, H. L. (1992). "Identification of soil properties from foundation impedance functions." Journal of Geotechnical Engineering, ASCE, 118(5), 780-795.

[66] Madera, G. (1971). "Fundamental period and amplification of peak acceleration in layered systems." Report No. R70-37, Dept. of Civil Eng., Massachusetts Institute of Technology, Cambridge, Massachusetts.

[67] A. Mohammad-Djafari, ed. (1998). Bayesian Inference for Inverse Problems, San Diego, California. SPIE.

[68] Najjar, Y. M., Basheer, I. A., and Ali, H. A. (1996). "Modeling stress-strain response of clay using neuro nets." Proceedings of the 11th-ASCE Speciality Conference on Engineering Mechanics, Y. K. Lin and T. C. Su, eds., ASCE, New York, NY. 697-700.

[69] Nazarian, S. (1984). "In situ determination of elastic moduli of soil deposits and pavement systems by spectral-analysis-of-surface-waves method," Ph.d thesis, The University of Texas at Austin.

[70] Nazarian, S. and Desai, M. R. (1993). "Automated surface wave method: Field testing." Journal of Geotechnical Engineering, ASCE, 119(7), 1094-1111.

[71] Nelles, O. (2001). Nonlinear System Identification: From Classical Approaches to Neural Networks and Fuzzy Models. Springer.

[72] Neuman, S. P. and Yakowitz, S. (1979). "A statistical approach to the problem of aquifer hydrology: 1. theory." Water Resources Research, 15, 845-860. 
[73] Newland, D. E. (1993). An Introduction to Random Vibrations, Spectral and Wavelet Analysis. Wiley, New York, 3rd edition.

[74] Ólafsson, S. and Sigbjornsson, R. (1995). "Application of arma models to estimate earthquake ground motion and structural response.” Earthquake Engng Struct. Dyn., 24, 951-966.

[75] Oskay, C. (2003). "Local identification analyses of soils and soil-structure systems," Ph.d thesis, Rensselaer Polytechnic Institute.

[76] Pavlenko, O. (2001). "Nonlinear seismic effects in soils: Numerical simulation and study." Bulletin of the Seismological Society of America, 91(2), 381-396.

[77] Peterka, V. (1981). "Bayesian system identification.” Automatica, 17(1), 123-162.

[78] Pintelon, R. and Schoukens, J. (2001). System Identification: A Frequency Domain Approach. John Wiley and Sons.

[79] Polhemus, N. W. and Cakmak, A. S. (1981). "Simulation of earthquake ground motions using autoregressive moving average (arma) models.” Earthquake Engng Struct. Dyn., 9, 343-354.

[80] Popescu, T. D. and Demetriu, S. (1990). "Analysis and simulation of strong earthquake ground motions using arma models." Automatica, 26(4), 721-737.

[81] Prevost, J. H. (1985). “A simple plasticity theory for frictional cohesionless soils.” Soil Dynamics and Earthquake Engineering, 4(1), 9-17.

[82] Ray, W. H. (1978). "Some recent applications of distributed parameter system theory: A survey." Automatica, 14, 281-287.

[83] Rix, G. J., Lai, C. G., and Spang Jr, A. W. (2000). "In situ measurement of damping ratio using surface waves." Journal of Geotechnical and Geoenvironmental Engineering, ASCE, 126(5), $472-480$.

[84] Safak, E. (1997). "Models and methods to characterize site amplification from a pair of records." Earthquake Spectra, 13, 97-129.

[85] Sayed, H. S. and Abdel-Ghaffar, A. M. (1992). "System identification of nonlinear soil response of earth dams." Proceedings of the 10th Conference on Earthquake Engineering, Vol. 8, Madrid, Spain. A. A. Balkema, 4733-4739.

[86] Shinozuka, M. and Ghanem, R. (1995). "Structural system identification ii: Experimental verification.” Journal of Engineering Mechanics, ASCE, 121(2), 265-273.

[87] Sidarta, D. E. and Ghaboussi, J. (1998). "Constitutive modeling of geomaterials from nonuniform material tests." Computers and Geotechnics, 22(1), 53-71.

[88] Skjærbæk, P. S., Kirkegaard, P. H., Foustikakis, G. N., and Fassois, S. D. (1997). "Non-stationary modeling and simulation of near-source earthquake ground motion: Arma and neural network methods." Proceedings of the XVth International Modal Analysis Conference. 1904-1910. 
[89] Steidl, J. H., Tumarkin, A. G., and Archuleta, R. J. (1996). "What is a reference site?” Bulletin of the Seismological Society of America, 86(6), 1733-1748.

[90] Stokoe, K. H., Nazarian, S., Rix, G. J., Salinero, I. S., Sheu, J. C., and Mok, Y. J. (1988). “In situ seismic testing of hard-to-sample soils by surface wave method." Earthquake Engineering and Soil Dynamics II- Recent Advances in Ground-Motion Evaluation, J. L. Von Thun, ed., Park City, Utah, 264-278.

[91] Taboada U., V. M., Martinez, H., Romo, M. P., and Ardila, C. D. (2000). "Identification of mexico city clay dynamic properties." Proceedings of the 12th World Conference on Earthquake Engineering, number 1220, New Zealand Society for Earthquake Engineering, Upper Hutt, New Zealand.

[92] M. Tanaka and G. S. Dulikravich, eds. (2000). Inverse problems in engineering mechanics, Nagano, Japan. Oxford, Elsevier Science.

[93] Tang, H. T. (1987). "Large scale soil structure interaction.” Report No. NP-5513-SR, Electric Power Research Institute, Palo Alto, California.

[94] Tarantola, A. (2005). Inverse Problem Theory and Methods for Model Parameter Estimation. Society for Industrial and Applied Mathematics (SIAM).

[95] Udwadia, F. E., Garba, J. A., and Ghodsi, A. (1984). "Parameter identification problems in structural and geotechnical engineering." Journal of Engineering Mechanics, ASCE, 110(9), 14091432.

[96] Unbehauen, H. and Rao, G. P. (1998). "A review of identification in continuous-time systems." Annual Reviews in Control, 22, 145-171.

[97] Wellstead, P. E. (1981). "Non-parametric methods of system identification.” Automatica, 17(1), 59-69.

[98] Wen, K.-L. (1994). "Non-linear soil response in ground motions." Earthquake Engng Struct. Dyn., 23, 599-608.

[99] Yang, J., Sato, T., and Li, X.-S. (2000). "Nonlinear site effects on strong ground motion at a reclaimed island." Canadian Geotechnical Journal, 37, 26-39.

[100] Yang, Z. and Elgamal, A. W. (2003). "Application of unconstrained optimization and sensitivity analysis to calibration of a soil constitutive model." International Journal for Numerical and Analytical Methods in Geomechanics, 27, 1277-1297.

[101] Yuan, D. and Nazarian, S. (1993). "Automated surface wave method: Inversion technique." Journal of Geotechnical Engineering, ASCE, 119(7), 1112-1126.

[102] Zeghal, M. and Abdel-Ghaffar, A. M. (1989). "Identification of the behavior of earth damsusing seismic records." Report CRECE 9001, Center for Research in EarthquakeEngineering and Construction, University of Southern California. 
[103] Zeghal, M. and Abdel-Ghaffar, A. M. (2009). "Evaluation of the nonlinear seismic response of earth dams: Nonparametric system identification." Journal of Earthquake Engineering, 13, 384405.

[104] Zeghal, M. and Elgamal, A. W. (1994). "Analysis of site liquefaction using earthquake records." Journal of Geotechnical Engineering, ASCE, 120(6), 996-1017.

[105] Zeghal, M. and Elgamal, A.-W. (2000). "Site response and vertical seismic arrays." Progress in structural Engineering and Materials, 2(1), 92-101. Invited article.

[106] Zeghal, M., Elgamal, A. W., and Parra, E. (1996). "Identification and modeling of earthquake ground response ii: Site liquefaction." Soil Dynamics and Earthquake Engineering, 15, 523-547.

[107] Zeghal, M., Elgamal, A. W., Tang, H. T., and Stepp, J. C. (1995). "Lotung downhole array. ii: Evaluation of soil nonlinear properties." Journal of Geotechnical Engineering, ASCE, 121(4), 363-378.

[108] Zeghal, M., Elgamal, A. W., Zeng, X., and Arulmoli, K. (1999). "Mechanism of liquefaction response in sand-silt dynamic centrifuge tests." Soil Dynamics and Earthquake Engineering, 18, $71-85$.

[109] Zeghal, M., Kallou, P. V., Oskay, C., Abdoun, T., and Sharp, M. K. (2006). "Visualization of soil and soil-structure response in the presence of liquefaction." Earthquake Engineering and Engineering Vibration, 5, 485-500.

[110] Zeghal, M. and Oskay, C. (2002). "Local system identification analyses of the dynamic response of soil systems.” Soil Dynamics and Earthquake Engineering, 22, 985-993.

[111] Zeghal, M. and Oskay, C. (2003). "A local identification technique for geotechnical and geophysical systems." International Journal for Numerical and Analytical Methods in Geomechanics, 27, 967-987.

[112] Zhu, J.-H., Zaman, M. M., and Anderson, S. A. (1998). "Modeling of soil behavior with a recurrent neural network." Canadian Geotechnical Journal, 35, 858-872.

[113] Zienkiewicz, O. C., Chan, A. H. C., Pastor, M., Schrefler, B. A., and Shiomi, T. (1998). Computational Geomechanics with Special Reference to Earthquake Engineering. John Wiley and Sons, England.

[114] Zorapapel, G. and Vucetic, M. (1994). “The effects of seismic pore water pressure on ground surface motion.” Earthquake Spectra, 10(2), 403-438. 